\title{
Life Cycle of Numerically Simulated Shallow Cumulus Clouds. Part I: Transport
}

\author{
Ming ZhaO* AND Philip H. Austin \\ Atmospheric Science Programme, Department of Earth and Ocean Sciences, University of British Columbia, Vancouver, \\ British Columbia, Canada
}

(Manuscript received 2 June 2003, in final form 1 September 2004)

\begin{abstract}
This paper is the first in a two-part series in which the life cycles of numerically simulated shallow cumulus clouds are systematically examined. The life cycle data for six clouds with a range of cloud-top heights are isolated from an equilibrium trade cumulus field generated by a large-eddy simulation (LES) with a uniform resolution of $25 \mathrm{~m}$. A passive subcloud tracer is used to partition the cloud life cycle transport into saturated and unsaturated components; the tracer shows that on average cumulus convection occurs in a region with time-integrated volume roughly 2 to 3 times that of the liquid-water-containing volume. All six clouds exhibit qualitatively similar vertical mass flux profiles with net downward mass transport at upper levels and net upward mass flux at lower levels. This downward mass flux comes primarily from the unsaturated cloud-mixed convective region during the dissipation stage and is evaporatively driven. Unsaturated negatively buoyant cloud mixtures dominate the buoyancy and mass fluxes in the upper portion of all clouds while saturated positively buoyant cloud mixtures dominate the fluxes at lower levels. Small and large clouds have distinct vertical profiles of heating/cooling and drying/moistening, with small clouds cooling and moistening throughout their depth, while larger clouds cool and moisten at upper levels and heat and dry at lower levels. The simulation results are compared to the predictions of conceptual models commonly used in shallow cumulus parameterizations.
\end{abstract}

\section{Introduction}

The importance of shallow cumulus convection for the redistribution of atmospheric heat and moisture is well established. Numerous approaches have been used to represent the mixing effect of these unresolved boundary layer clouds in large-scale models, including eddy diffusivity approximations, moist convective adjustment schemes, and mass flux parameterizations (e.g., Tiedtke et al. 1988; Betts 1986; Arakawa and Schubert 1974). Mass flux parameterizations are the most sophisticated of these approaches; they are built around specific conceptual models that constrain the way in which clouds can interact with the environment. Examples of such conceptual models include the entraining plume model (e.g., Stommel 1947; Arakawa and Schubert 1974), episodic mixing and buoyancy sorting models (EMBS; e.g., Raymond and Blyth 1986; Kain and Fritsch 1990; Emanuel 1991; Emanuel and

\footnotetext{
* Current affiliation: NOAA/Geophysical Fluid Dynamics Laboratory, Princeton, New Jersey.
}

Corresponding author address: Dr. Ming Zhao, NOAA/ Geophysical Fluid Dynamics Laboratory, Princeton University, Forrestal Campus, Box 308, U.S. Route 1, Princeton, NJ 08542. E-mail: ming.zhao@noaa.gov
Zivkovic-Rothman 1999), and the transient cumulus model of von Salzen and McFarlane (2002).

All of these mass flux parameterizations must account either implicitly or explicitly for the size distribution of the unresolved clouds. For example, Arakawa and Schubert (1974) represent the cloud population by a spectrum of entraining plumes with different entrainment rates or, equivalently, cloud-top heights. Implicit representations of the cloud size distribution model the net effect of the cloud population as a single entraining/ detraining plume with variable entrainment and detrainment rates (Kain and Fritsch 1990; Siebesma and Holtslag 1996). The free parameters for all of these schemes can be adjusted to reproduce the average vertical mass flux profile as inferred from measurements of the large-scale forcing in statistical steady state.

An obvious drawback to tuning the parameterized mass flux profile to observations is that it limits the conditions under which the parameterization can be used. If the size spectrum and the conceptual cloud model can be considered independently, it becomes possible to introduce new observational constraints on the parameterization. As Grinnell et al. (1996) point out, the conceptual models listed above make distinct predictions for both the vertical mass transport within individual clouds, and the manner in which this transport varies with height due to cloud entrainment and 
detrainment. An entraining plume model predicts increasing mass transport with height due to lateral entrainment, with all detrainment taking place at cloud top. In an EMBS model, both entrainment and detrainment take place at all heights within the cloud layer so that mass transport may actually decrease with height above cloud base. Transient cumulus models introduce the cumulus life cycle into the bulk representation of the entraining and detraining plume so that the lifetime-averaged cloud fraction and mass flux may also decrease with height.

The EMBS models also differ in their treatment of the detrainment of mixed cloud air into the environment. For example, the EMBS scheme of Emanuel (1991, hereafter E91) detrains cloud mixtures into their unsaturated neutral buoyancy levels (UNBL), defined as the neutral buoyant level (NBL) obtained by reversible adiabatic evaporation of the detraining parcel's liquid water content. This detrainment criterion essentially defines the cloud boundary to encompass the evaporating mixed region so that the evaporation directly cools the cloud air (Emanuel 1994). Transmission of this cooling to the environment is realized through environmental compensating upward motion induced by cloud downdrafts. In contrast, the EMBS model of Raymond and Blyth (1986), in common with entraining plume models, directly detrains liquid water into the environment. A similar approach is used by Kain and Fritsch (1990), who detrain neutral and negatively buoyant saturated mixtures directly into the environment. Both profiles of vertical mass flux and the cloud heating and moistening rates are sensitive to this detrainment criterion (see, e.g., Zhao and Austin 2003).

Direct observations of the vertical profile of vertical mass flux within cumulus clouds can help distinguish between these competing conceptual models. Doppler radar, used in concert with in situ aircraft observations, provides one tool for examining the vertical mass flux profile over the life cycle of individual clouds. Grinnell et al. (1996) combined two ground-based Doppler radars and an instrumented aircraft to compute the vertical mass flux in trade wind cumulus clouds during the Hawaiian Rainband Project of 1990. They found that, averaged over the cloud's lifetime, the vertical profile of net vertical mass flux was negative in the upper portion of the cloud layer and positive at lower levels. The cloud life cycle was characterized by a growth phase in which the mass flux was upward at all levels, a mature stage in which there was downward mass flux at upper levels, and a dissipation stage during which the mass flux was everywhere downward.

A complementary approach is provided by largeeddy simulations (LES) and cloud-resolving models, which have been increasingly used to study cumulus convective transport (e.g., Tao et al. 1987; Siebesma and Cuijpers 1995; Lin and Arakawa 1997; Brown et al. 2002; Stevens et al. 2001). Siebesma and Cuijpers (1995), for example, found that the vertical profiles of both cloud cover and cloud vertical mass flux for the Barbados Oceanographic and Meteorological Experiment (BOMEX) trade cumulus boundary layer was maximum near cloud base and decreased monotonically to zero with height, indicating numerous small clouds. This feature of shallow convection is common to 10 different LES codes (Siebesma et al. 2003). The LES-determined cloud size distribution has also been studied by Neggers et al. (2003), who found consistency between the modeled cloud field and satellite observations.

The examination of the individual clouds embedded in a simulated cloud ensemble has until recently received little attention. There have been numerous simulations of single cumulus clouds developing in a conditionally unstable atmosphere (e.g., Klaassen and Clark 1985; Grabowski and Clark 1993; Bretherton and Smolarkiewicz 1989). However, the simulated structure and time evolution of these clouds depend on the "trigger" that releases the stored energy and therefore may be sensitive to the size and shape of the initial impulse. This is in general inappropriate in low-buoyancy environments such as shallow convection (Carpenter et al. 1998). In principle, this ad hoc specification of the cloud environment and initialization can be overcome by studying individual clouds embedded in LES runs that are independent of initial conditions. There is reason to think that individual shallow clouds may be realistically simulated in such models. For example, Siebesma and Jonker (2000) show that the fractal dimension of the simulated individual cloud boundaries at $100-\mathrm{m}$ resolution are in excellent agreement with observations. As mentioned above, Neggers et al. (2003) retrieved cloud size distributions from several LES datasets at 50-m resolution and found them to be consistent with satellite observations. These models appear to have reached the stage where they can resolve both the cloudensemble statistics and some of the individual cloud dynamics.

In this paper, we use an LES model run to examine the life cycle of the vertical cloud mass flux, with an emphasis on studying the effects of unsaturated convection within the cloud-mixed region using passive tracers. We use a set of six clouds to examine the role of cloud size in the cloud ensemble transport. Our objective is to test some of the physical assumptions underlying conceptual models of cumulus clouds used in cumulus parameterizations. In a companion paper (Zhao and Austin 2005, hereafter Part II) we focus on cloud mixing dynamics for these same six clouds. In section 2 we briefly describe the LES model, the simulation setup, and the approach used to isolate and identify individual clouds and cloud-mixed convective regions. In section 3 we present the simulated individual clouds and their life cycles with emphasis on the vertical transport of mass, the role of buoyancy in convective mass flux, and thermodynamic transport based on conditional sampling of cloud mixtures. In section 4 we test 
the sensitivity of these results to the choice of threshold used to identify the unsaturated convective mixed region. We further discuss the model results and their implications for conceptual models of cumulus mixing in section 5 .

\section{Approach}

\section{a. The LES model and case description}

The Colorado State University System for Atmospheric Modeling (SAM) is used in this study; a detailed description of this model is given in Khairoutdinov and Randall (2003). The equations of motion are written using the anelastic approximation. The subgridscale model employs a 1.5-order closure based on the prognostic subgrid-scale turbulent kinetic energy. The advection of momentum is computed with secondorder finite differences while all prognostic scalar variables are advected using the fully three-dimensional positive definite and monotonic scheme of Smolarkiewicz and Grabowski (1990). Periodic boundary conditions are applied for both east-west and north-south lateral boundaries; the top boundary is treated with a sponge layer to damp the spurious reflection of upward propagating gravity waves, while the bottom boundary uses prescribed sensible and latent heat flux as will be presented below. We use a three-dimensional domain with $256 \times 256 \times 128$ grid points with uniform grid spacing $\Delta x=\Delta y=\Delta z=25 \mathrm{~m}$ and a model time step of $1.5 \mathrm{~s}$.

We choose the undisturbed trade wind boundary layer from Phase III of the BOMEX. A detailed description of the LES initialization can be found in Siebesma et al. (2003), together with statistics from 10 LES models, including an earlier version of SAM. Statistics for the current SAM version are presented in Zhao (2003); the performance of SAM on the BOMEX simulation is well represented by the envelope of model results reported in Siebesma et al. (2003).

The convection in the boundary layer is driven by a specified clear air radiative cooling rate and surface heat fluxes, with latent and sensible heat fluxes of $8 \times$ $10^{-3} \mathrm{~K} \mathrm{~m} \mathrm{~s}^{-1}$ and $5.2 \times 10^{-5} \mathrm{~m} \mathrm{~s}^{-1}$, respectively. Figures $1 \mathrm{a}-\mathrm{c}$ show the initial sounding profiles for the Global Energy and Water Cycle Experiment (GEWEX) Cloud Systems Study (GCSS) BOMEX intercomparison simulation. There is a well-mixed subcloud layer between approximately 0 and $500 \mathrm{~m}$, a conditionally unstable cloud layer between 500 and $1500 \mathrm{~m}$, and an overlying inversion layer. The wind profiles are initialized as easterly with north-south component $v$ set to zero. Figures $1 \mathrm{~d}-\mathrm{f}$ show the prescribed forcings, which include large-scale subsidence, clear-sky radiative cooling, and advective drying in the lower subcloud layer. Although idealized, the GCSS BOMEX case setup ensures a consistent set of large-scale forcings that establish an equilibrium for a shallow cumulus
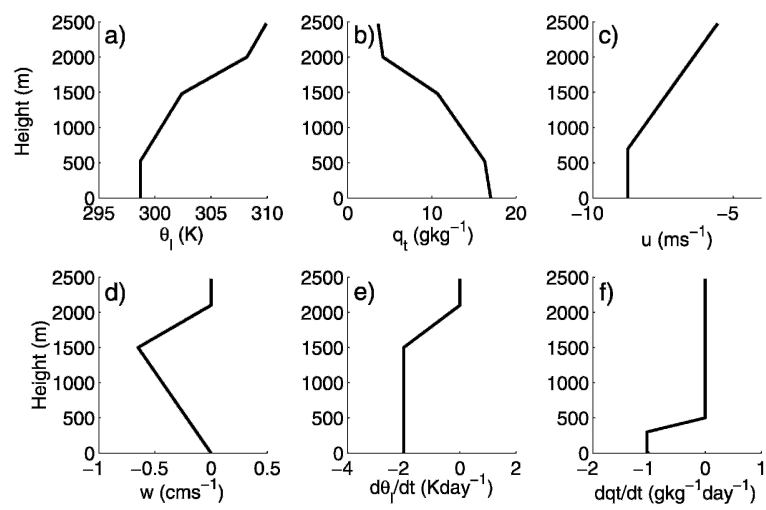

FIG. 1. Initial sounding and large-scale forcing profiles for the LES BOMEX case (see text for details). (a) Liquid water potential temperature $\theta_{l}$, (b) total water mixing ratio $q_{t}$, (c) horizontal wind (only have east-west component $u$ while north-south component $v$ is initialized as zero), (d) large-scale subsidence, (e) clear-air radiative cooling, and (f) large-scale advective drying.

cloud boundary layer in the LES. The model-produced equilibrium vertical profiles of temperature and moisture closely match the observed BOMEX atmosphere (Nitta and Esbensen 1974; Siebesma and Cuijpers 1995). The first $3 \mathrm{~h}$ of the simulation are considered to be spinup, in which the system reaches its steady-state equilibrium. We run the LES for $6 \mathrm{~h}$; all selected clouds are sampled between hours 3 and 6 .

\section{b. Isolating individual clouds}

Since the BOMEX environment has strong easterly ambient wind (Fig. 1c) the simulated individual clouds move rapidly from east to west. Therefore the distance that an individual cloud travels during its life cycle is much larger than its horizontal size. To isolate individual clouds over their life cycle and obtain timedependent five-dimensional cloud data we must sample the cloud in a reference frame that moves to the west at approximately the mean horizontal velocity. The coordinate in the moving frame $x_{\text {new }}=x-x_{0}(t)=x-U_{0} t$, where $x$ is the coordinate in the fixed frame and $x_{0}(t)=$ $U_{0} t$ is the coordinate of the origin of the moving frame in the fixed frame. We choose a translation velocity $U_{0}$ $=-7.5 \mathrm{~m} \mathrm{~s}^{-1}$, which is close to the horizontally and vertically averaged mean velocity within the cloud layer. Consistent with the LES, periodic boundary conditions are used when we apply the translation.

The time-sampled cloud field moves much more slowly in the translated reference frame, allowing us to use fixed boxes to contain individual clouds throughout their life cycle. Note that the Galilean invariance of the Navier-Stokes equations ensures that the behavior of the fluid is unchanged in this new inertial reference frame (e.g., Pope 2000). During the isolation process we always try to select a box that includes a single cloud with as much cloud-free surrounding environment as possible. In this study we examine six clouds with cloud- 
top heights (defined as the maximum height reached by cloud liquid water) ranging from 1000 to $2000 \mathrm{~m}$. They are independent of each other in both time and space and will be referred to below (ordered smallest to largest) by the labels A, B, C, D, E, and F.

\section{c. Distinguishing the convective region from the environment}

To help with the isolation of individual clouds, and more importantly to provide an objective way to identify the cloud-mixed convective region (which may be unsaturated), we use a passive numerical tracer, with mass mixing ratio $\zeta$, initialized to 0 above cloud base and $1 \mathrm{~g} \mathrm{~kg}^{-1}$ below cloud base. We first run the LES, obtain a particular realization of the time-dependent cloud fields, and select individual clouds. Since we now know the start and end times and the cloud-base height of each cloud, we rerun the LES with the tracer initialized at a single time step just before an individual cloud emerges from the subcloud layer.

Only advection ${ }^{1}$ is applied to the subcloud layer tracer; the tracer variable aids in tracking the destination of subcloud air based on the resolved velocity. In this simulation, cumulus convection is the only process that can bring subcloud air to appreciably higher levels. Dry thermals may also overshoot the cloud-base height for a short distance and bring some tracer to levels slightly above cloud base. Although the overshooting dry thermals always sink back after entraining some environmental air near the cloud-base level, they do contaminate the time-dependent cloud data near cloud base. In addition, implicit numerical diffusion due to the advection scheme may also transport tracer across the sharp tracer interface near the cloud-base level. Therefore, data sampled near the cloud-base level must be interpreted with caution.

Given the isolated individual clouds $\mathrm{A}-\mathrm{F}$, the mixed region (MR) associated with each cloud will be defined as those grid cells above cloud-base level that initially contained no subcloud layer tracer $\zeta$ but now contain $\zeta$ $>\zeta_{0}$ due to the cloud upward transport and mixing of subcloud air with its upper environment. Here $\zeta_{0}$ is a threshold value of the subcloud layer tracer mixing ratio. Ideally, $\zeta_{0}$ should be chosen so that the MR defined by $\zeta>\zeta_{0}$ includes all the liquid water containing grid cells [i.e., grid cells with $q_{c}>0$, which will be called liquid water cloud (LWC) below]. Figure 2 shows an example of the joint frequency distribution (JFD) of $\zeta$ and $q_{c}$ for all LWC grid cells during the lifetime of cloud E (a similar pattern holds for the other clouds).

\footnotetext{
${ }^{1}$ A simulation with both advection and subgrid-scale diffusion applied to the subgrid-scale tracer produces little difference. This is due to the fact that the current advection scheme (Smolarkiewicz and Grabowski 1990) includes an effective subgrid-scale model when no explicit turbulence is applied, as has been demonstrated in Margolin et al. (1999).
}

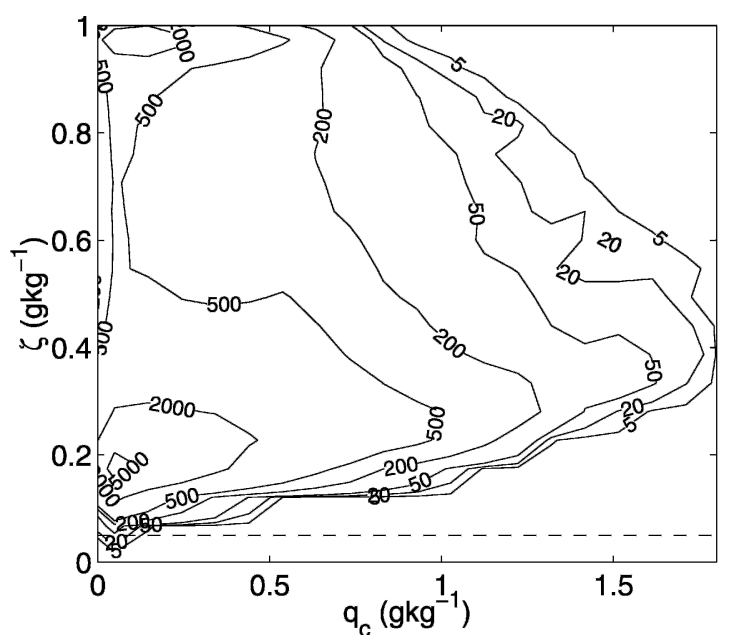

FIG. 2. Joint frequency distribution of subcloud layer tracer mixing ratio $\zeta$ and liquid water mixing ratio $q_{c}$ for all liquid-watercontaining grid cells during the life cycle of cloud $\mathrm{E}$. The contoured unit is number of grid cells. The dashed line indicates the $\zeta_{0}=0.05 \mathrm{~g} \mathrm{~kg}^{-1}$ threshold.

As the figure shows, for $\zeta_{0}>0.05 \mathrm{~g} \mathrm{~kg}^{-1}$ the MR defined by $\zeta>\zeta_{0}$ begins to exclude some LWC grid cells. Therefore we choose $\zeta_{0}=0.05 \mathrm{~g} \mathrm{~kg}^{-1}$ for all six selected clouds; with this threshold the sampled MR includes nearly all $(>99 \%)$ of the LWC. This choice of tracer level is conservative in the sense that it selects the smallest MR, which includes nearly all the LWC.

The criterion $\zeta>\zeta_{0}$, however, does not guarantee that the MR is convective since some grid cells in the MR may simply contain detrained subcloud layer air that has finished its convective circulation and mixing and finally reached equilibrium with the stratified environment. Figure 3 shows the JFD of $w$ and $\Delta \theta_{v}$ within the unsaturated MR $\left(\zeta>\zeta_{0}\right.$ and $\left.q_{c}=0\right)$ for each cloud over its life cycle. Here, $\Delta \theta_{v}=\theta_{v}-\left\langle\theta_{v}\right\rangle, \theta_{v}$ is the grid cell value and angle brackets represent a horizontal model domain average. The figure shows that the distribution mode is centered on $w=0$ and $\Delta \theta_{v}=0$, values that correspond to the quiescent environment. Based on this distribution we define a detrained mixed region (DMR) as all grid cells that satisfy the criterion $\left(\zeta>\zeta_{0}\right.$ and $q_{c}=0$ and $\left|\Delta \theta_{v}\right| \leq \Delta \theta_{v, 0}$ and $\left.|w| \leq w_{0}\right)$, where $\Delta \theta_{v, 0}$ and $w_{0}$ are adjustable positive threshold values. Given the DMR, the convective mixed region (CMR), which is the focus of this paper, can be defined as CMR $=$ MR - DMR. The CMR defines the convective envelope associated with the individual cumulus transport and includes both the LWC and the unsaturated convective mixed region (UCMR); that is, CMR $=\mathrm{UCMR}+\mathrm{LWC}$. Table 1 summarizes these five conditionally sampled regions, while Fig. 4 gives a schematic illustration of their position as well as the location of overshooting thermals near cloud base. As discussed above, these dry thermals do transport tracer and produce some grid cells near cloud base, which may be misclassified as part 

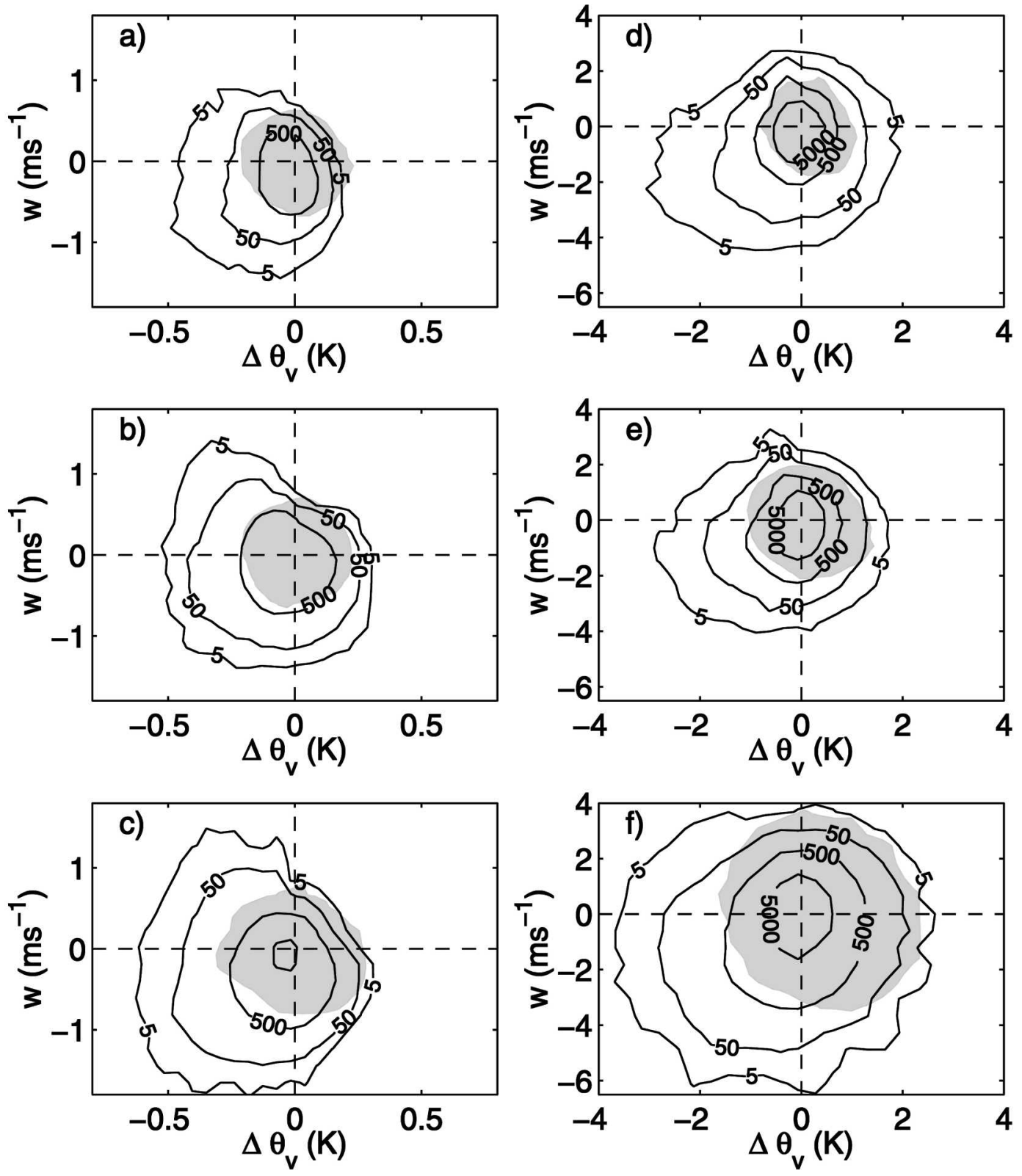

FIG. 3. Contours represent joint frequency distribution of $\Delta \theta_{v}$ and $w$ within the unsaturated cloud-mixed region (defined as $q_{c}=0 \mathrm{~g} \mathrm{~kg}^{-1}$ and $\zeta>0.05 \mathrm{~g} \mathrm{~kg}^{-1}$ ) for clouds A-F [(a)-(f) respectively]. Shaded region represents the range of $\Delta \theta_{v}$ and $w$ values that encompass more than $99.6 \%$ of the unmixed grid cells, defined as $q_{c}=0 \mathrm{~g} \mathrm{~kg}^{-1}$ and $\zeta<0.01 \mathrm{~g} \mathrm{~kg}^{-1}$. Percentages for shaded regions are (a) $99.64 \%$, (b) $99.86 \%$, (c) $99.84 \%$, (d) $99.94 \%$, (e) $99.95 \%$, and (f) $99.96 \%$.

of the MR. This misclassified MR volume makes a negligible contribution to the mass flux a short distance (200 $\mathrm{m})$ above the cloud-base level.

Choosing large values for $\Delta \theta_{v, 0}$ and $w_{0}$ selects a larger DMR and hence a smaller and more active UCMR; arbitrarily large $\Delta \theta_{v, 0}$ and $w_{0}$ will eliminate all the UCMR and reduce the sampled CMR to LWC only. In principle, the choice of $\Delta \theta_{v, 0}$ and $w_{0}$ may depend on the variability of the unmixed environment's $\Delta \theta_{v}$ and $w$. The shaded region on each panel of Fig. 3 shows the set of grid cell $\left(\Delta \theta_{v}, w\right)$ values that characterize at least $99.6 \%$ of the grid cells in the unmixed environment (i.e., those grid cells with $q_{c}=0$ and $\zeta<0.01 \mathrm{~g} \mathrm{~kg}^{-1}$ ). For the smaller clouds (Figs. $3 \mathrm{a}-\mathrm{c}$ ), these environment grid cells lie approximately within the range $\left|\Delta \theta_{v}\right|<0.2$ $\mathrm{K},|w|<0.5 \mathrm{~m} \mathrm{~s}^{-1}$ ). For the larger clouds (Figs. 3d-f) the environment spans a much larger range of $\Delta \theta_{v}, w$ (note the different scales between the left and right panels). This larger environmental variation is limited to the inversion levels; beneath the inversion the envi- 
TABLE 1. Definition of the conditionally sampled regions. Note that $\mathrm{CMR}=\mathrm{LWC}+\mathrm{UCMR}$ and $\mathrm{MR}=\mathrm{CMR}+\mathrm{DMR}$.

\begin{tabular}{ll}
\hline \hline \multicolumn{1}{c}{ Name } & \multicolumn{1}{c}{ Definition } \\
\hline MR (mixed region) & $\begin{array}{c}\text { all grid cells above cloud-base } \\
\text { level with } \zeta>\zeta_{0}\end{array}$ \\
DMR (detrained mixed region) & $\zeta>\zeta_{0}$ and $q_{c}=0$ and \\
& $\left|\Delta \theta_{v}\right| \leq \Delta \theta_{v, 0}$ and $|w| \leq w_{0}$ \\
CMR (convective mixed region) & MR but not DMR \\
$\begin{array}{c}\text { UCMR (unsaturated convective } \\
\text { mixed region) }\end{array}$ & CMR and $q_{c}=0$ \\
$\begin{array}{c}\text { LWC (liquid water cloud) } \\
\text { CMR and } q_{c}>0\end{array}$ \\
\hline
\end{tabular}

ronmental $\left(\Delta \theta_{v}, w\right)$ fluctuations have roughly the same range in all clouds (not shown). Although clouds that penetrate the inversion generate internal gravity waves causing large fluctuations in the environmental $\theta_{v}$ and $w$ at upper levels, these waves make a negligible contribution to the net vertical mass transport.

Below we use, for simplicity, the height-independent thresholds that apply below the inversion $\left(\Delta \theta_{v, 0}=0.2 \mathrm{~K}\right.$ and $w_{0}=0.5 \mathrm{~m} \mathrm{~s}^{-1}$ ) for all levels. While we expect these threshold choices to underestimate the volume of the DMR within the inversion for large clouds, we show in section 4 that this has relatively little impact on the UCMR mass flux within the inversion. Choosing a larger threshold, however, produces a significant underestimate of the UCMR mass flux below the inversion. Note that the $\left(\zeta, w, \Delta \theta_{v}, q_{c}\right)$ criteria have to be simultaneously satisfied in order to identify a grid cell that will not become convective. For example, a neutrally buoyant region with zero vertical velocity may still be potentially convective if it has liquid water since further mixing with the subsaturated environment may generate negative buoyancy and, therefore, downward velocity.

\section{Results}

\section{a. Life cycle overview}

Figure 5 shows the top and base height of each cloud over its lifetime, together with the maximum updraft and downdraft velocity. The cloud top and base height at each time step are determined by the highest and lowest levels that contain liquid water. Small clouds A, $\mathrm{B}$, and $\mathrm{C}$ have a maximum height around $1000-1400 \mathrm{~m}$ and do not reach the inversion layer (which spans 1500$2000 \mathrm{~m}$ and is rather weak for the BOMEX). Clouds D, $\mathrm{E}$, and $\mathrm{F}$ are large clouds, which penetrate into the inversion. In contrast to their very different cloud-top heights, all clouds have essentially the same cloud-base heights, which do not vary with time prior to the dissipation stage. The uniform cloud-base height is due to the homogeneous heat flux specified over the ocean surface in this simulation. Individual cloud lifetimes end when all liquid water is evaporated (possibly before all the unsaturated cloud mixtures reach their NBL). Lifetime animations (available online at http:// www.eos.ubc.ca/research/clouds; Zhao and Austin 2004)] of these clouds show that small clouds A, B, and $\mathrm{C}$ contain a single ascending updraft that decays after reaching its maximum height, while large clouds D, E, and $\mathrm{F}$ tend to contain two to three pulselike updrafts. Each succeeding pulse ascends and decays similarly, but reaches a maximum height lower than its predecessor. Because of these multiple pulses, large clouds D, E, and $\mathrm{F}$ have longer lifetimes $(\tau \approx 25 \mathrm{~min})$ compared with small clouds $(\tau \approx 18 \mathrm{~min})$.

Figures $5 \mathrm{c}, \mathrm{d}$ show the time evolution of the maximum updraft and downdraft velocities within individual clouds. The maximum upward velocity of each cloud

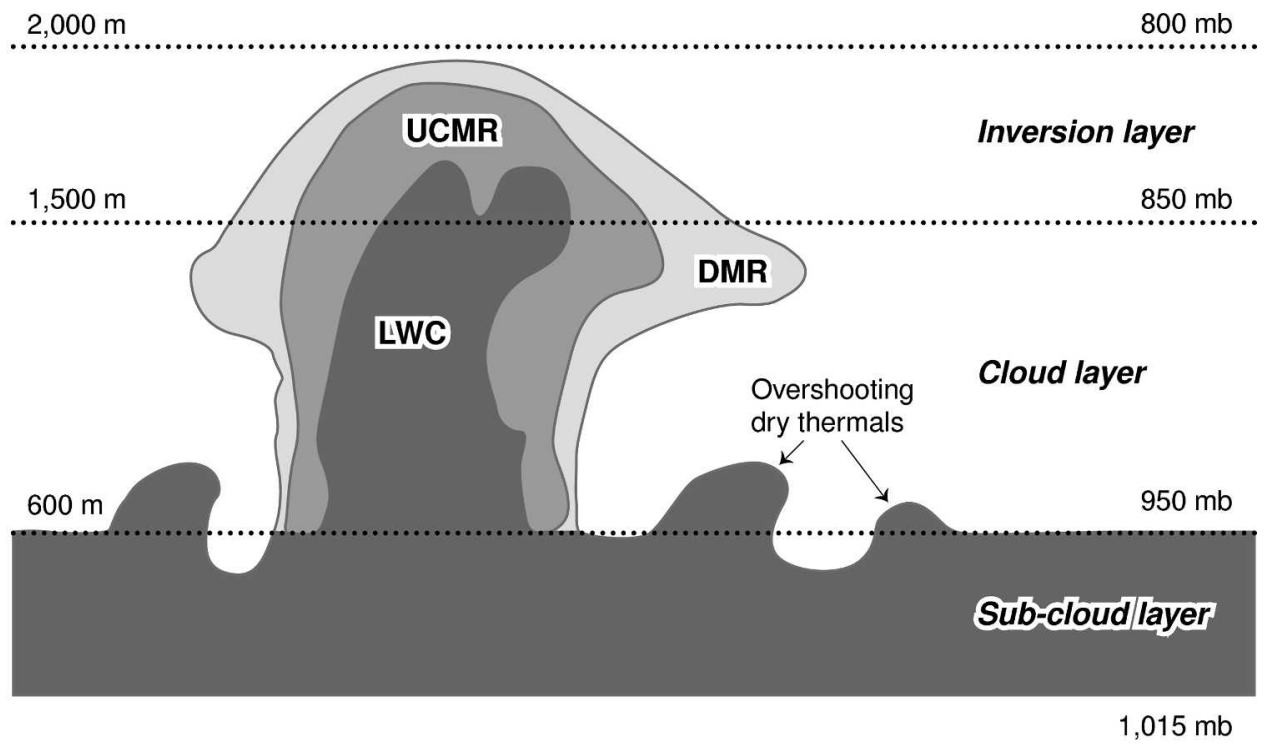

FIG. 4. Schematic illustration of three conditionally sampled regions defined in Table 1. 

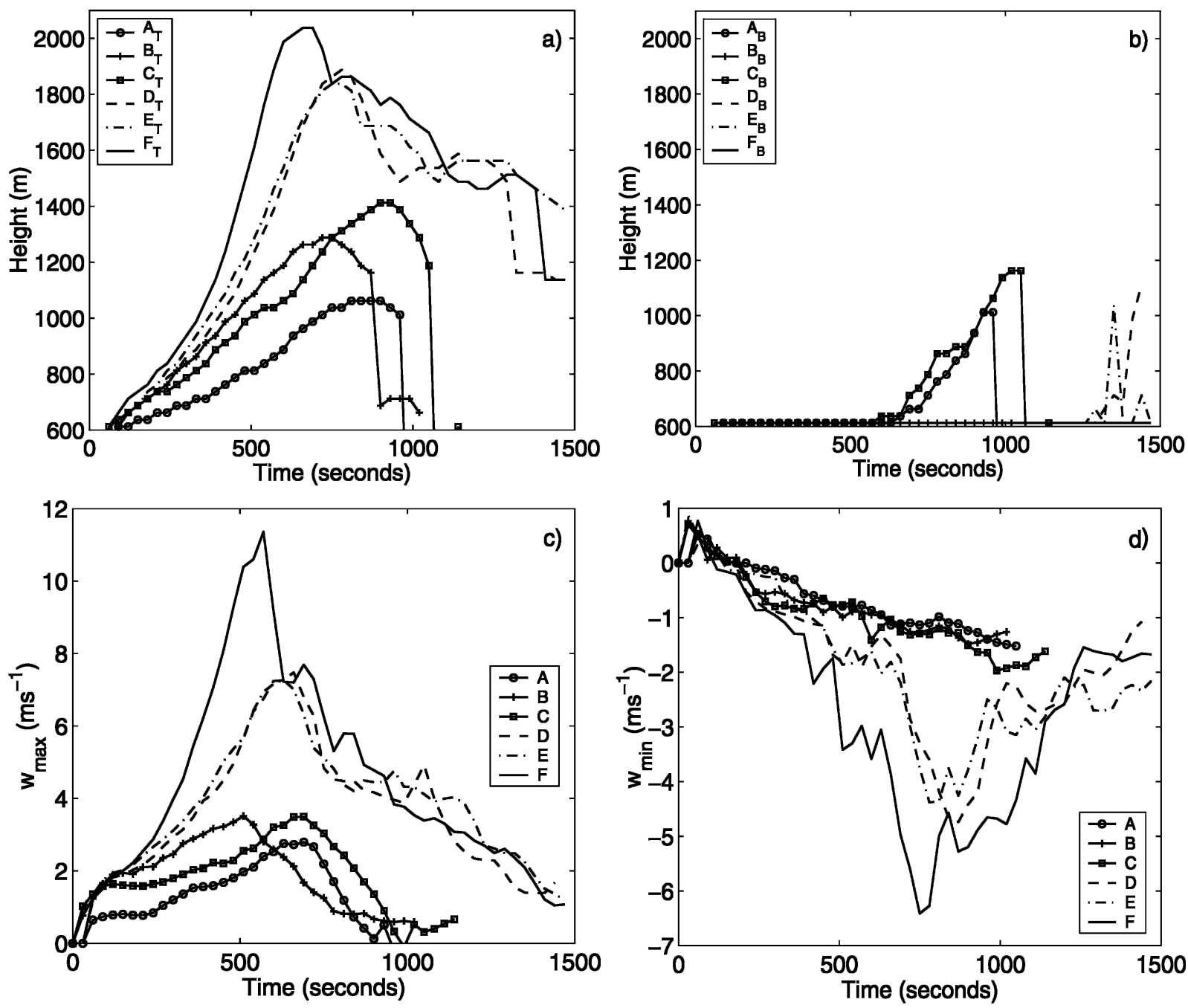

Fig. 5. Life cycles of clouds A-F. (a) Cloud-top heights (subscript T), (b) cloud-base heights (subscript B), (c) maximum upward velocities, and (d) maximum downward velocities.

always occurs before arrival at the maximum height, while the strongest downdraft occurs slightly after arrival at the maximum height. This pattern is associated with the penetration of the ascending cloud tops above their NBLs, where the cloud tops decelerate as they continue to rise. The collapse of cloud top and the associated mixing and evaporation triggers the strongest downdrafts. During the developing stage of each cloud the height associated with $w_{\max }$ is always well correlated with the cloud-top height; that is, it is always several hundred meters below the cloud top-environment interface. A detailed examination of individual clouds indicates that the differences among the similar sizes of clouds (A, B and C as small clouds; D, E, and F as large clouds) are much smaller than those between small and large clouds. Therefore, below we average separately across the small clouds $\mathrm{A}, \mathrm{B}$, and $\mathrm{C}$ and large clouds D, $\mathrm{E}$, and $\mathrm{F}$ and present the averaged results of small and large clouds.
Figures $6 \mathrm{a}, \mathrm{b}$ show the time evolution of the volumes of the LWC and UCMR for small and large clouds. During the cloud ascending stage the UCMR is smaller than the LWC for all clouds. By the time the clouds reach their maximum height, the UCMRs have volumes that are roughly half (small clouds) or about twothirds (large clouds) of their LWC volumes. After the clouds reach their maximum height, the LWC volumes begin to shrink due to continuous evaporation, while the UCMRs continue to increase due to turbulent mixing. A short time before the cloud liquid water is completely evaporated, the UCMRs reach their maximum volume and begin to rapidly decrease. This decrease is due to the exclusion of mixtures that have reached equilibrium with the environment based on the $\left(\Delta \theta_{v, 0}\right.$, $w_{0}$ ) thresholds. When the evaporation of the liquid water content is nearly complete, the UCMRs are still roughly as large as the maximum LWC. This indicates that the lifetime of individual clouds as defined by the 

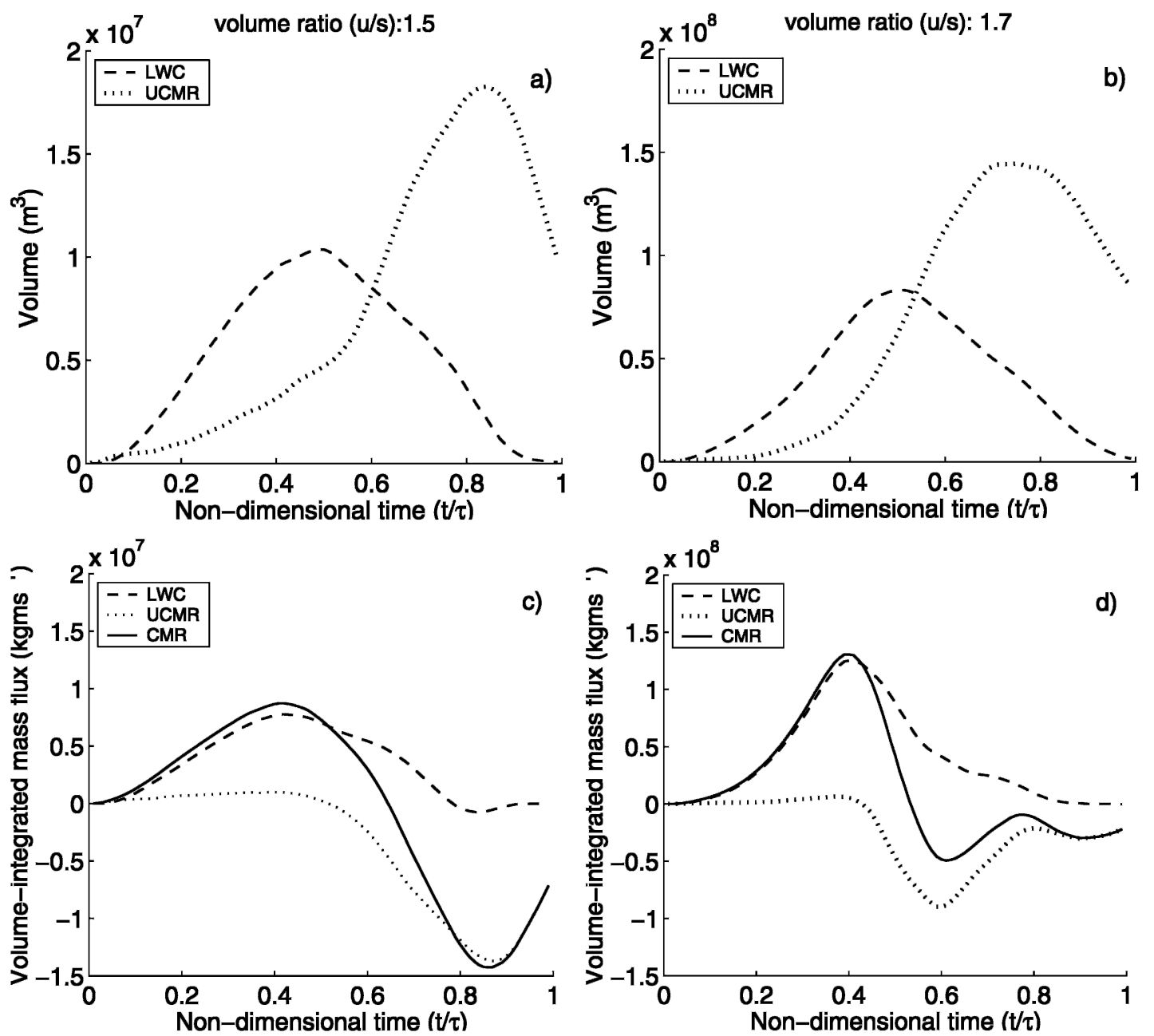

FIG. 6. (a) Time evolution of the LWC and UCMR volumes averaged over small clouds A, B, and C. Time is nondimensionalized by individual cloud lifetime. The number at the top of each panel shows the ratio of the timeintegrated volume of UCMR and LWC. (b) As in (a) but averaged over large clouds D, E, and F (note the different scale). (c) Time evolution of the volume-integrated vertical mass flux for the LWC, UCMR, and CMR averaged over small clouds A, B, and C. (d) As in (c) but averaged over large clouds D, E, and F.

existence of liquid water tends to be slightly shorter than the convective period associated with the turbulent mixing of individual clouds. Integration over individual cloud lifetimes reveals that the volume of the total CMR is about 2 to 3 times the size of the LWC (see the ratios at the top of Figs. 6a,b). Translating these volumes to equivalent cylindrical radii implies that the horizontal radius of the total CMR is roughly 1.5 times larger than the radius of the visible cloud (LWC).

Figures $6 \mathrm{c}, \mathrm{d}$ show the time evolution of the vertical mass flux integrated over the two regions of Figs. 6a,b. For each cloud the mass flux is generally positive (upward) in the LWC and negative (downward) in the UCMR with flux in the LWC dominating the earlier phase, while the flux in the UCMR dominates the later phase of convection. Figures $6 c, d$ show that there is a transition in the total volume-integrated vertical mass flux from positive to negative beginning at approximately one-half of the cloud lifetime with a roughly oscillatory time variation. Integrated over the cloud life cycle, this produces a negative net vertical mass flux for small clouds and a positive net vertical mass flux for large clouds.

\section{b. Vertical profiles of the lifetime-averaged vertical mass flux}

Figures $7 \mathrm{a}, \mathrm{b}$ show the vertical profiles of cloud lifetime-averaged vertical mass flux for the small and large clouds. All clouds have net downward mass flux in their upper levels. The downward mass flux for small clouds extends deeper into the cloud layer while for large inversion-penetrating clouds the net downward mass flux is limited to approximately the upper one-third of the cloud depth, producing net downward mass flux within the inversion. The cloud-top downward mass flux of the 

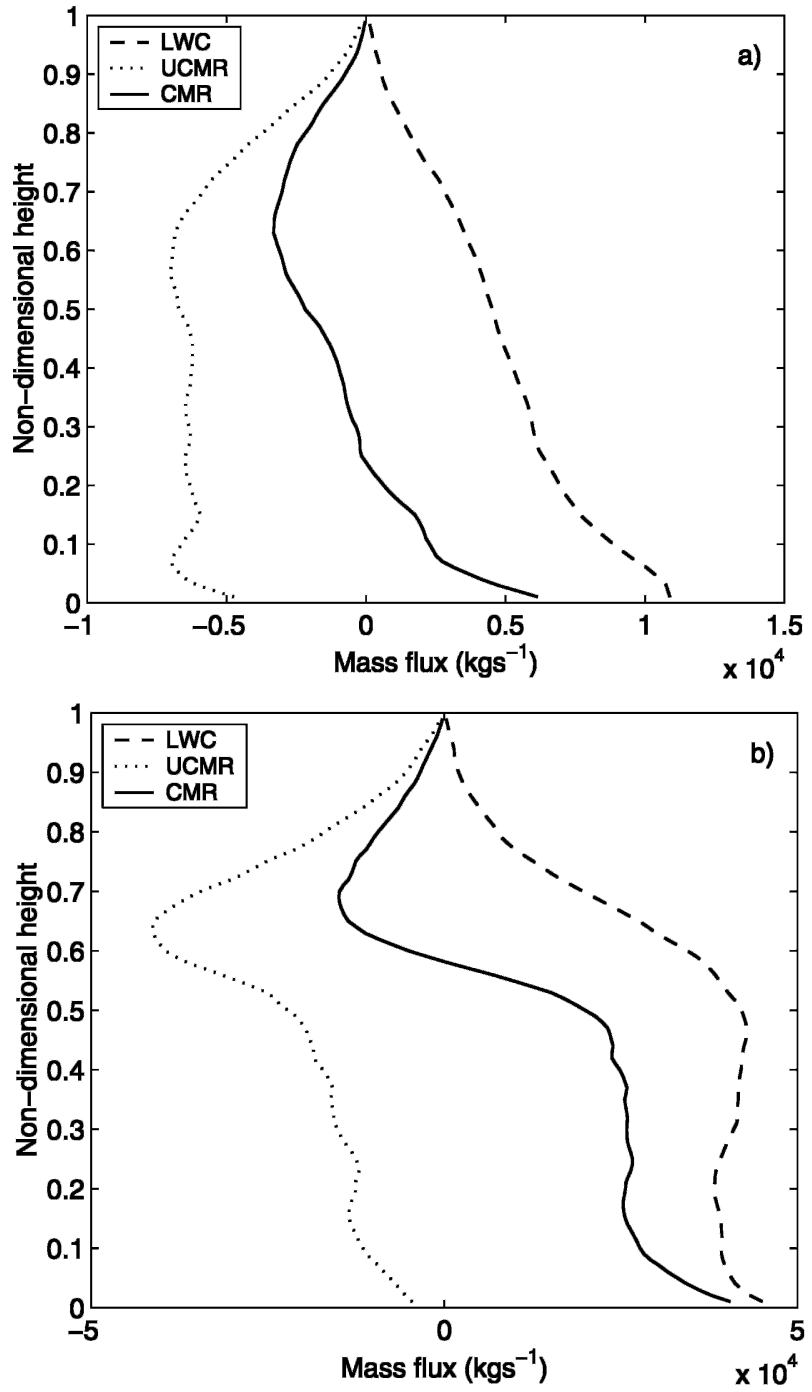

FIG. 7. (a) Vertical profiles of the lifetime-averaged vertical mass flux for LWC, UCMR, and CMR averaged over small clouds $\mathrm{A}, \mathrm{B}$, and $\mathrm{C}$. Height is nondimensionalized by individual cloud height. (b) As in (a) but averaged over large clouds D, E, and F.

simulated clouds is consistent with the aircraft and radar observations of Hawaiian trade cumuli reported by Raga et al. (1990) and Grinnell et al. (1996). It is also consistent with the result of Zhao and Austin (2003, hereafter ZA03), who diagnosed the cloud ensemble vertical mass flux using an EMBS model based on the BOMEX mean soundings and large-scale forcings. The partitioned vertical profiles of vertical mass flux in Figs. $7 \mathrm{a}, \mathrm{b}$ reveal that the downward mass flux comes primarily from the UCMR while the LWC produces, on average, upward mass flux throughout the cloud depth (although a significant number of saturated downdrafts do exist near cloud top). In section 5 we discuss the implications of this vertical profile of vertical mass flux for the shallow cumulus parameterization problem.

A life cycle view of the vertical distribution of verti- cal mass flux is shown in Fig. 8. The vertical mass fluxes integrated over the horizontal CMR at different vertical levels and time steps are contoured and shaded in the time-height space for clouds A-F. As the figure shows, during the developing stage all clouds produce net upward mass flux. When the clouds reach their mature stage (i.e., cloud tops reach their maximum heights) the downward mass flux begins to increase. Net downward mass flux occurs first near cloud top for the large clouds (D, E, and F), while for small clouds, downward mass flux appears first at lower levels, before the cloud tops reach their maximum heights. During the dissipation stage all clouds have net downward mass flux throughout their depth. The magnitudes of the downward mass fluxes are as large as those of the upward mass fluxes. Thus, at every level, the time history of the vertical mass flux resembles the time variation of Figs. $6 c, d$; near cloud base the upward flux during the growth phase is larger than the downward flux at the dissipation stage, while near cloud top this pattern is reversed. In general, this life cycle of the vertical profile of the cloud vertical mass flux through the growth and dissipation stages is again consistent with the radar observations of Grinnell et al. (1996), who found negative net mass flux in the upper portion of the cloud layer at the dissipation stage and positive mass flux at lower levels during the growth phase for small nonprecipitating Hawaiian clouds.

\section{c. The role of buoyancy in vertical mass transport}

The buoyancy-sorting hypothesis suggests that cloud mixtures coming from above an observation level should have negative or neutral buoyancy at the observation level, while mixtures arriving from below should have positive or neutral buoyancy. In other words, cloud mixtures tend to move following their buoyancy and produce a positive buoyancy flux. To test the buoyancy-sorting hypothesis with these simulated clouds, we next evaluate the contribution of "buoyancy direct" and "buoyancy indirect" mass fluxes to the convective mass transport. Here we define buoyancy-direct motion as vertical motion that is positively correlated with the buoyancy; buoyancy-indirect or counterbuoyancy transport is characterized by a negative velocitybuoyancy correlation.

Figure 9a shows the JFD of $\left(q_{t}, \Delta \theta_{v}\right)$ over all CMR grid cells at a level within the inversion $(1612.5 \mathrm{~m}$ above the surface) over the life cycle of cloud E. Note the strong correlation between mixture buoyancy and the total water mixing ratio $q_{t}$, which, as a conserved variable, is usually well correlated with the mixture's cloud-environment mixing fraction. The square symbol marks the $\left(q_{t}, \Delta \theta_{v}\right)$ of cloud-base air lifted adiabatically to this level, while the circle represents the $\left(q_{t}, \Delta \theta_{v}\right)$ of the mean environment. The well-defined $\mathrm{V}$ shape indicates the strong nonlinear dependence of the mixture buoyancy on mixing fraction caused by phase change. The quadrants separate mixtures in the CMR into four 

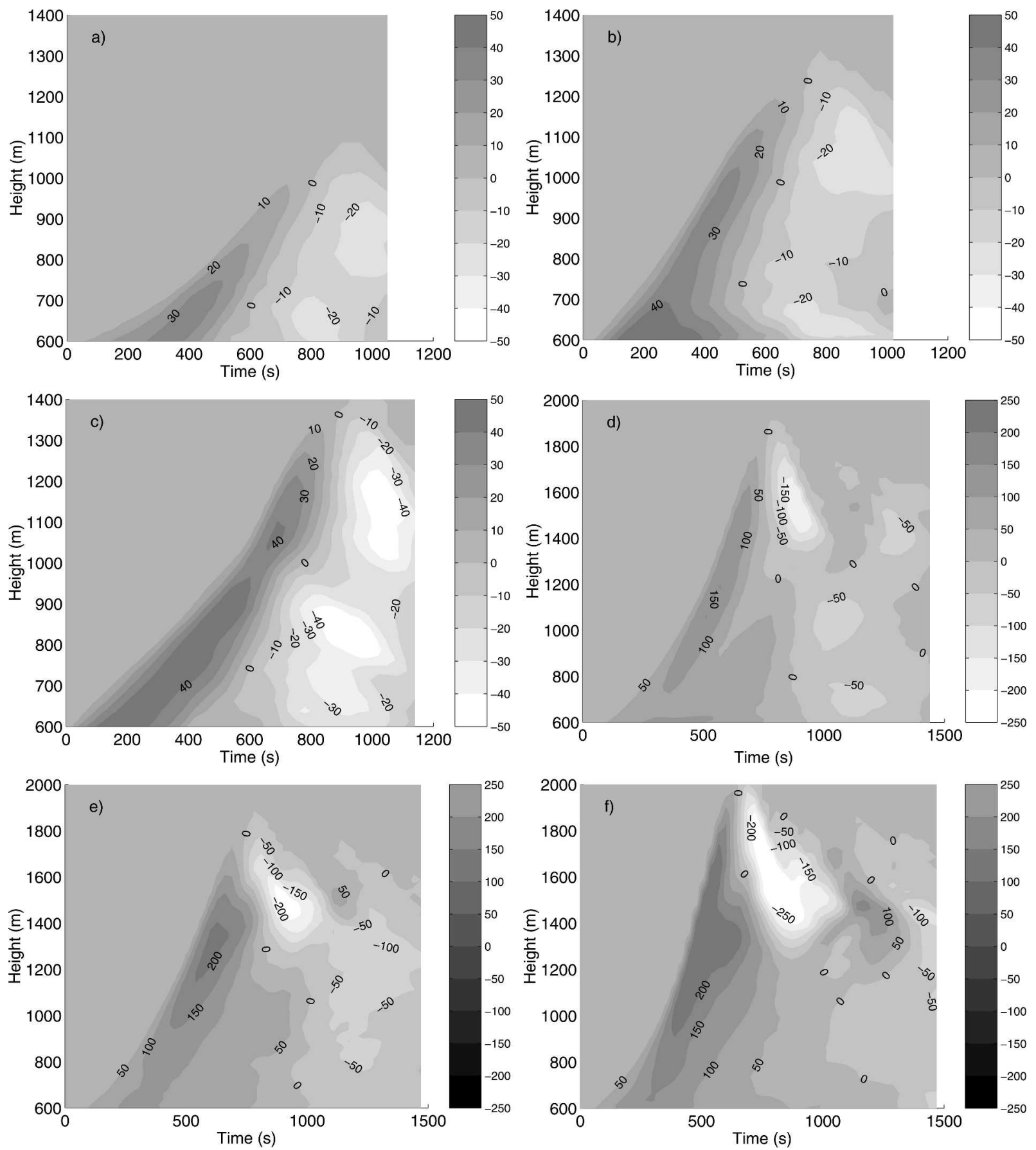

FIG. 8. Time-height variation of the vertical mass flux [i.e., contours of $\left(\int_{A} \rho w d A\right)_{t, k}$ (unit: $\left.1000 \mathrm{~kg} \mathrm{~s}^{-1}\right)$ for time step $t$, vertical level $k$ ] integrated over the horizontal CMR area $A$ for clouds A-F [(a)-(f), respectively].

categories: saturated positively buoyant (SP), saturated negatively buoyant (SN), unsaturated negatively buoyant (UN), and unsaturated positively buoyant (UP).

Figure $9 \mathrm{~b}$ shows a contour plot of the bin-averaged vertical velocity $\hat{w}$ using the same grid cells and times of Fig. 9a. There is both buoyancy-direct and buoyancyindirect motion, with unsaturated mixtures dominating the downward motion, while saturated mixtures dominate the upward motion. Figures 10a,b show the partitioned vertical profile of the vertical mass flux for the four different categories averaged over small and large clouds. The SP mixtures tend to dominate the upward mass flux, with maxima peaking near cloud base, while UN mixtures tend to dominate the downward mass 

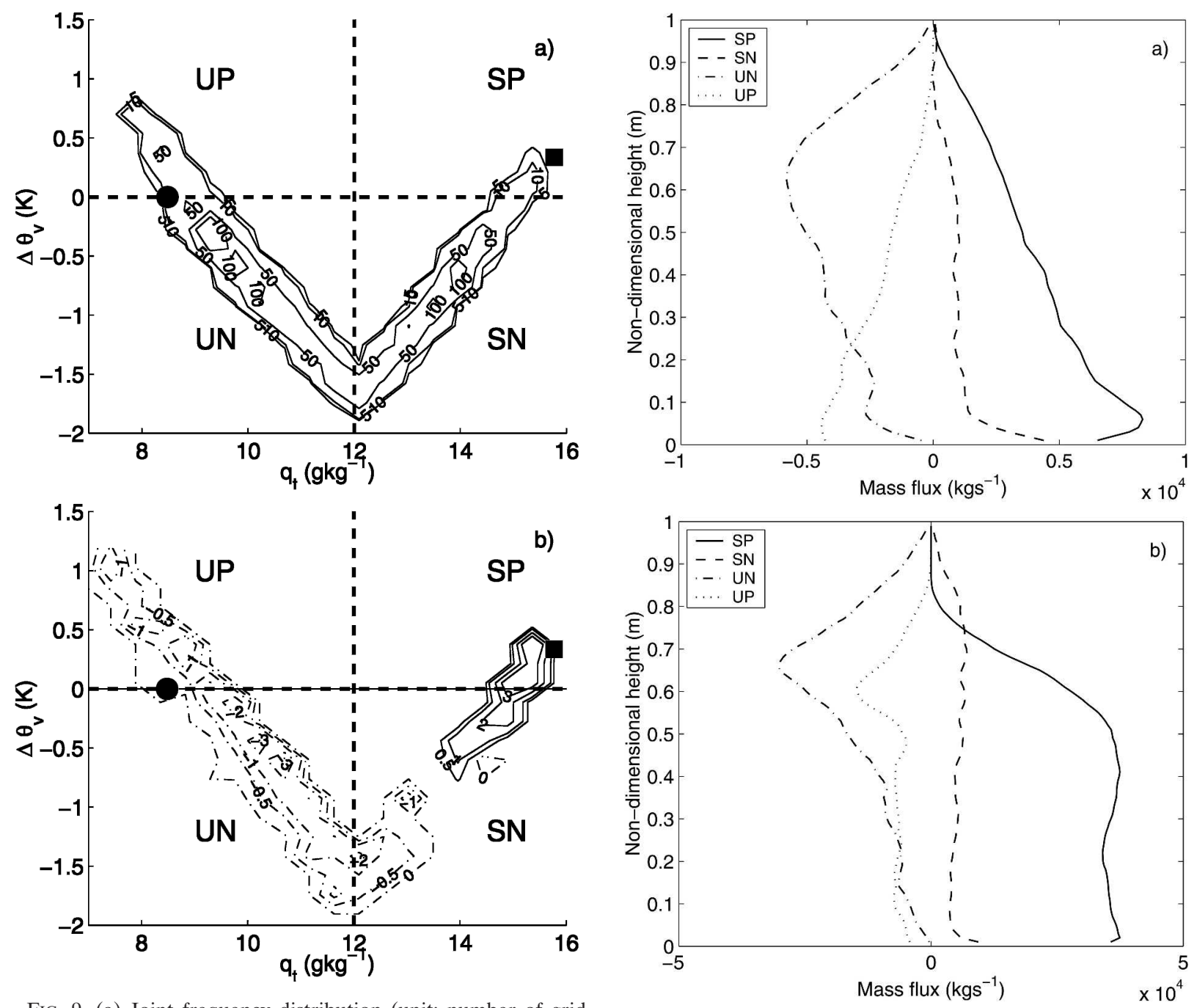

FIG. 9. (a) Joint frequency distribution (unit: number of grid cells) of $\Delta \theta_{v}$ and $q_{t}$ for the CMR at a single level (1612.5 m, $250 \mathrm{~m}$ below the maximum cloud top of $1867.5 \mathrm{~m}$ ) over the life cycle of cloud E. The quadrants separate (right) saturated from (left) unsaturated, (up) positively buoyant from (down) negatively buoyant mixtures. The square symbol represents cloud-base air lifted adiabatically to this level, while the circle represents the mean environment at this level. (b) Bins as in (a) but contours are the bin-averaged vertical velocity $\hat{w}\left(\mathrm{~m} \mathrm{~s}^{-1}\right)$. Dashed contours: downward $\hat{w}$; solid contours: upward $\hat{w}$.

flux, with maxima peaking near cloud top. Both are buoyancy direct. However, significant counterbuoyancy transport also occurs. In particular, Fig. 10 shows that SN mixtures, on average, transport mass upward and UP mixtures, on average, transport mass downward.

The JFD of $\Delta \theta_{v}$ and $w$ within the LWC for each cloud during its lifetime is shown in Fig. 11. While most of the SP mixtures have positive vertical velocity, this is not true for SN mixtures. In fact, the majority of the SN mixtures in Fig. 11 have positive vertical velocity. This mixture component can be produced by a mixing process in which momentum exchange allows a mixture to

FIG. 10. (a) Vertical profiles of the vertical mass flux partitioned into contributions from four mixture categories of Fig. 9. These profiles are averaged over small clouds A, B, and C. (b) As in (a) but the averaged over large clouds $\mathrm{D}, \mathrm{E}$, and $\mathrm{F}$.

maintain positive velocity while evaporation produces buoyancy reversal. An additional source for these upward-moving, negatively buoyant mixtures are initially positively buoyant mixtures carried past their NBL by their inertia. For either case, these mixtures will not return to their initial NBL if mixing continues along their counterbuoyant trajectories. Figure 9 shows that there are many more unsaturated downdrafts than saturated downdrafts for cloud $\mathrm{E}$; this is true for all six of the simulated clouds, indicating that further mixing and phase change is a dominant feature of the counterbuoyancy transport.

In contrast, the UN mixtures do not experience this phase change, and the UP mixtures are generated primarily by initially UN mixtures overshooting their NBL from above. As will be shown in section 3d, these UP 

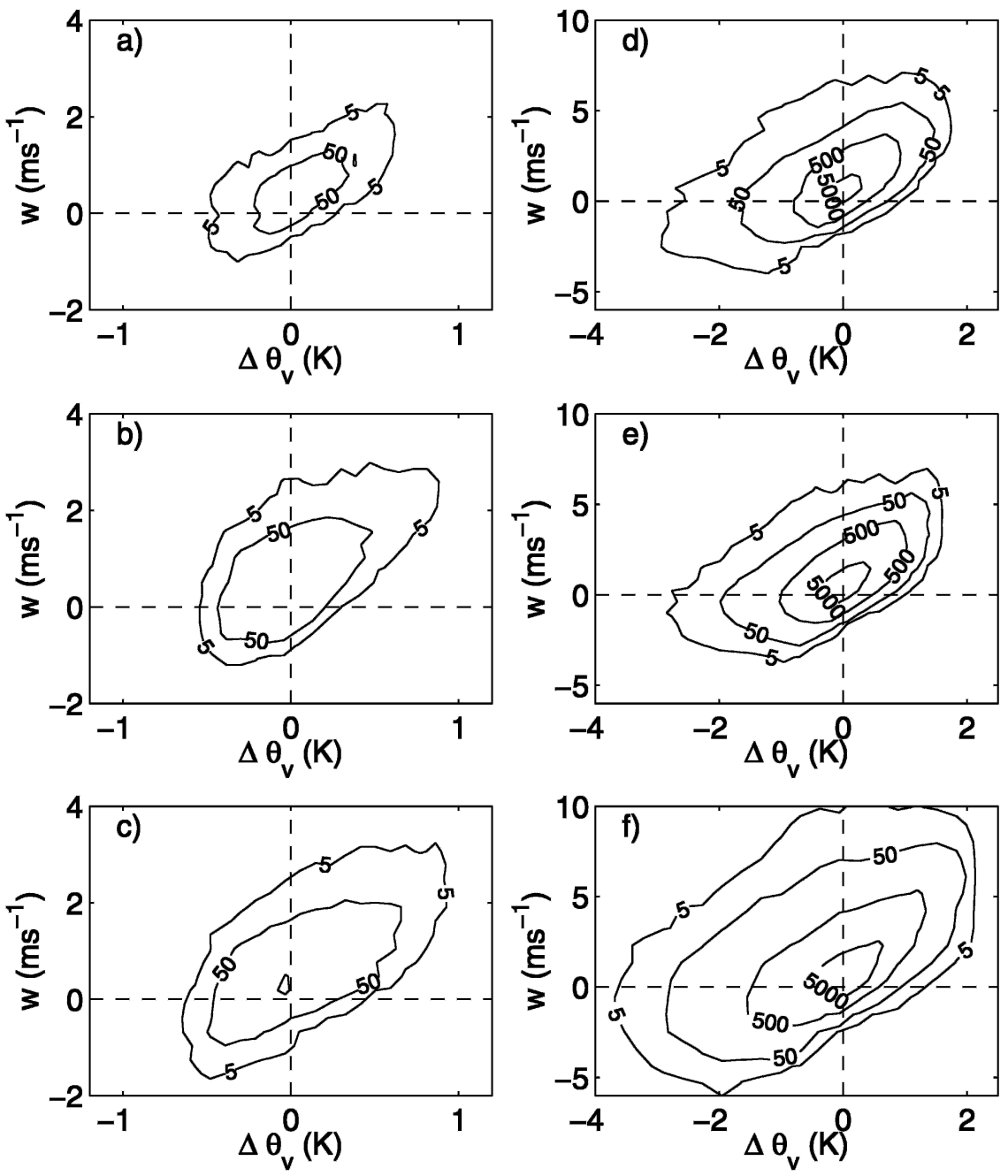

FIG. 11. Joint frequency distribution (unit: number of grid cells) of $\Delta \theta_{v}$ and $w$ within the LWC for clouds A-F [(a)-(f), respectively].

mixtures typically have thermodynamic properties very close to their environment as they reach their NBL and therefore transport negligible amounts of heat and moisture.

Figure 12 summarizes our discussion of the role of buoyancy in vertical mass transport. It shows the buoyancy flux partitioned into buoyancy-direct (BD) and buoyancy-indirect (BI) components. In general, the BD component dominates the overall convective mass flux and produces positive buoyancy flux throughout the individual cloud depths, supporting the buoyancysorting hypothesis. However, significant BI (counterbuoyancy) transport does exist, particularly near cloud base for small clouds and near cloud top for large clouds. This counterbuoyancy transport is primarily associated with mixture inertia and indicates that a buoyancy-sorting model that transports all cloudenvironment mixtures based solely on their buoyancy may potentially over-estimate the buoyancy flux. This is in contrast to the tendency of entraining plume models to underestimate the buoyancy flux, as shown by Siebesma and Cuijpers (1995). The relatively large counterbuoyancy transport at cloud base in Fig. 12a is caused by a combination of saturated updrafts that experience negative buoyancy (i.e., cloud inhibition) near the cloud base and unsaturated downdrafts that overshoot their NBL from above.

\section{d. The nature of the unsaturated downdrafts}

As shown in Fig. 10 the unsaturated negatively buoyant mixtures dominate the downward mass flux. More information about the thermodynamic properties of the cloud-environment mixtures is given in Fig. 13, which is a mixing diagram using $\left(\theta_{l}, q_{t}\right)$ coordinates following Taylor and Baker (1991). Values in the scatterplot are taken from all grid cells at the 1612.5 -m inversion level over the lifetime of cloud E (cf. Fig. 9). These mixtures 

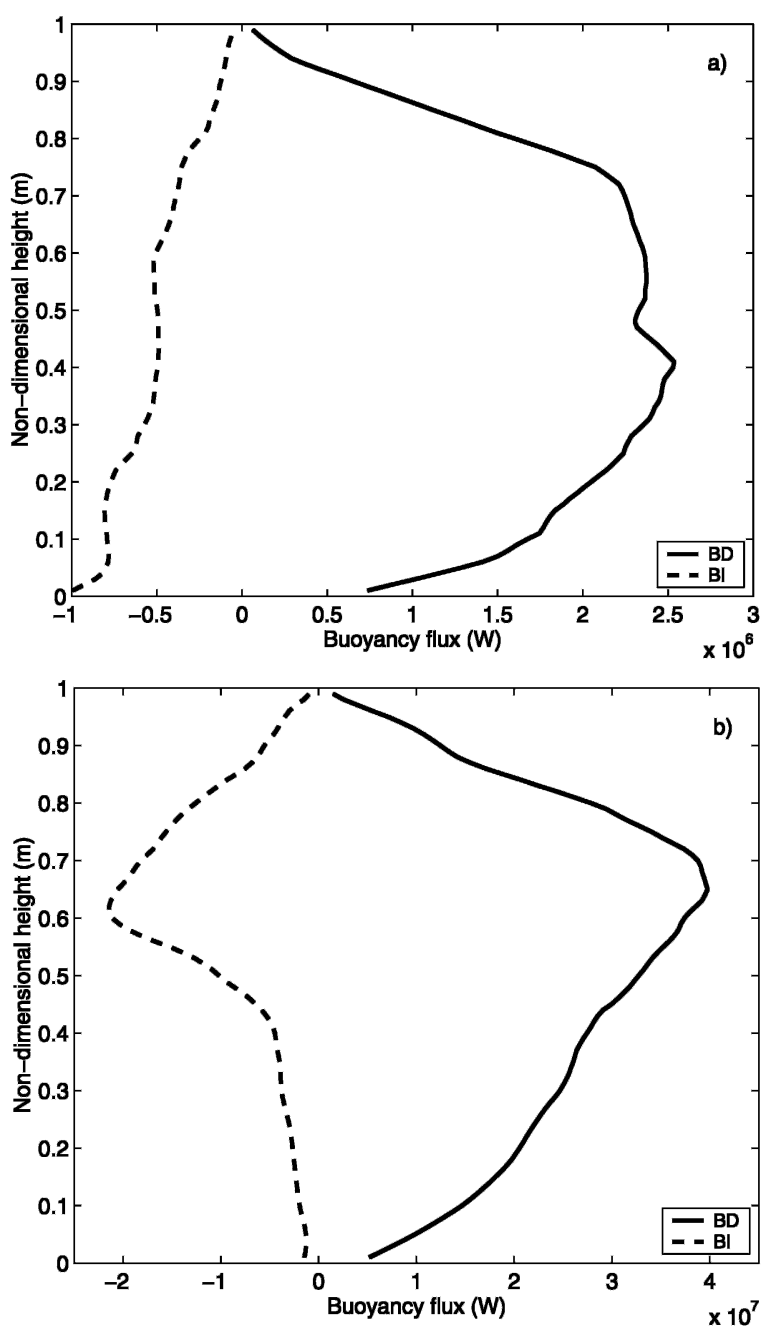

FIG. 12. Vertical profiles of the buoyancy flux partitioned into buoyancy-direct (BD) and buoyancy-indirect (BI) components. Panels (a) and (b) are averaged over small clouds (A-C) and large clouds (D-F), respectively.

are distributed along a mixing line between the thermodynamic coordinates of cloud-base environmental air (symbol: $\times$ ) and those for air at (or 100 to $200 \mathrm{~m}$ above) the current observation level (symbol: + ). This linear mixing line between cloud-base air and air at, or slightly above, the observation level is a feature of numerous in situ observations in cumulus clouds.

Also shown on the diagram are the average environmental sounding (solid line), $\theta_{v}$ isopleths at $1612.5 \mathrm{~m}$ (dash-dot), and the saturation line at $1612.5 \mathrm{~m}$ (dashed). As the figure shows, nearly $50 \%$ of the mixture range at this level is unsaturated; these mixtures have negative buoyancy of the same magnitude as their saturated counterparts. These unsaturated mixtures are often neglected in analyses of in situ aircraft measurements and numerical simulations. The continuous character of this mixing distribution, however, underscores

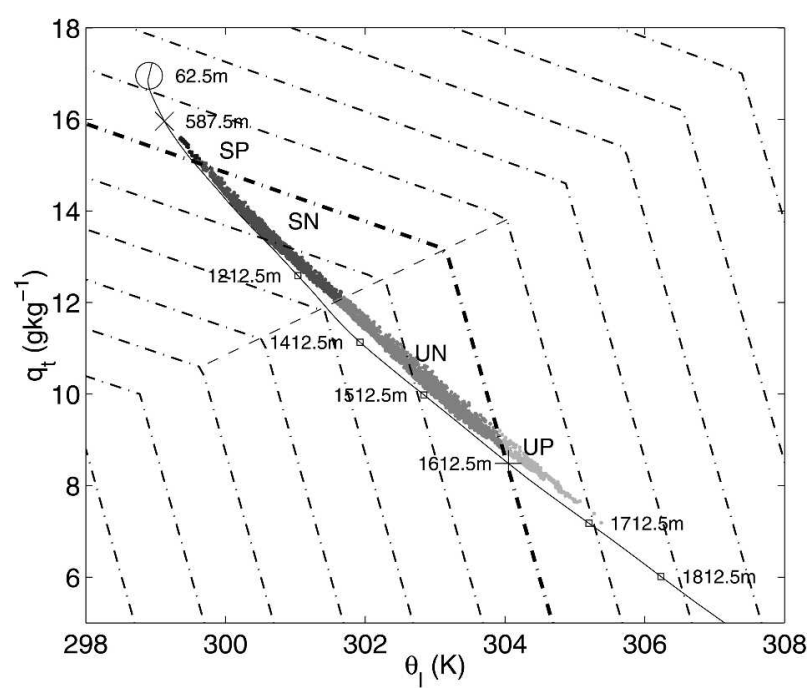

FIG. 13. Convective cloud mixtures plotted on a $\left(\theta_{l}, q_{t}\right)$ conserved variable diagram. Points: all CMR grid cells at the 1612.5-m level during the lifetime of cloud $\mathrm{E}$, shaded to denote the four mixture categories, labeled as in Fig. 9. Solid line: the vertical profile of environmental $\left(\theta_{l}, q_{t}\right)$, with the values at 1612.5 $\mathrm{m}$ indicated by $(+)$ and values at other height by $\square$. The circle indicates the surface layer mean properties, while the $(x)$ indicates the cloud-base mean properties. Thin dash-dot lines: $\theta_{v}$ isopleths for air at this level. Thick dash-dot line: the zero buoyancy isopleth $\left(\theta_{v}=305.6 \mathrm{~K}\right)$ at this level. Dashed line: the saturation curve.

the fact that unsaturated mixtures are an integral part of cumulus mixing throughout the cloud life cycle.

Figures 14 show partitioned lifetime-averaged vertical profiles of the $\theta_{l}$ and $q_{t}$ difference between each mixture category and the environment. The partitioning of the four categories is as in Fig. 9. As noted above and in section $3 \mathrm{c}$, the UP mixtures for all clouds do have $\theta_{l}$ and $q_{t}$ nearly indistinguishable from their environment, supporting the idea that they are primarily due to UN mixtures overshooting their NBLs. Figure 14 also shows that the UN mixtures, which are primarily responsible for the downward mass flux, are on average systematically cooler and moister than the environment. This again indicates that they must be associated with cloud mixing and evaporation, since downdrafts that were instead purely mechanically forced by saturated updrafts would be drier and warmer than the surrounding environment.

\section{e. Thermodynamic fluxes and tendencies}

Figures $15 \mathrm{a}-\mathrm{d}$ show the fluxes of two conserved variables, $\theta_{l}$ and $q_{t}$, partitioned into the four mixture categories as in Fig. 10. The $\theta_{l}$ and $q_{t}$ fluxes produced by SN and UN mixtures always have opposite sign and tend to cancel throughout the cloud depth, while the $\theta_{l}$ and $q_{t}$ flux contributions due to UP mixtures are small throughout the cloud depth. Thus it is the SP mixtures 

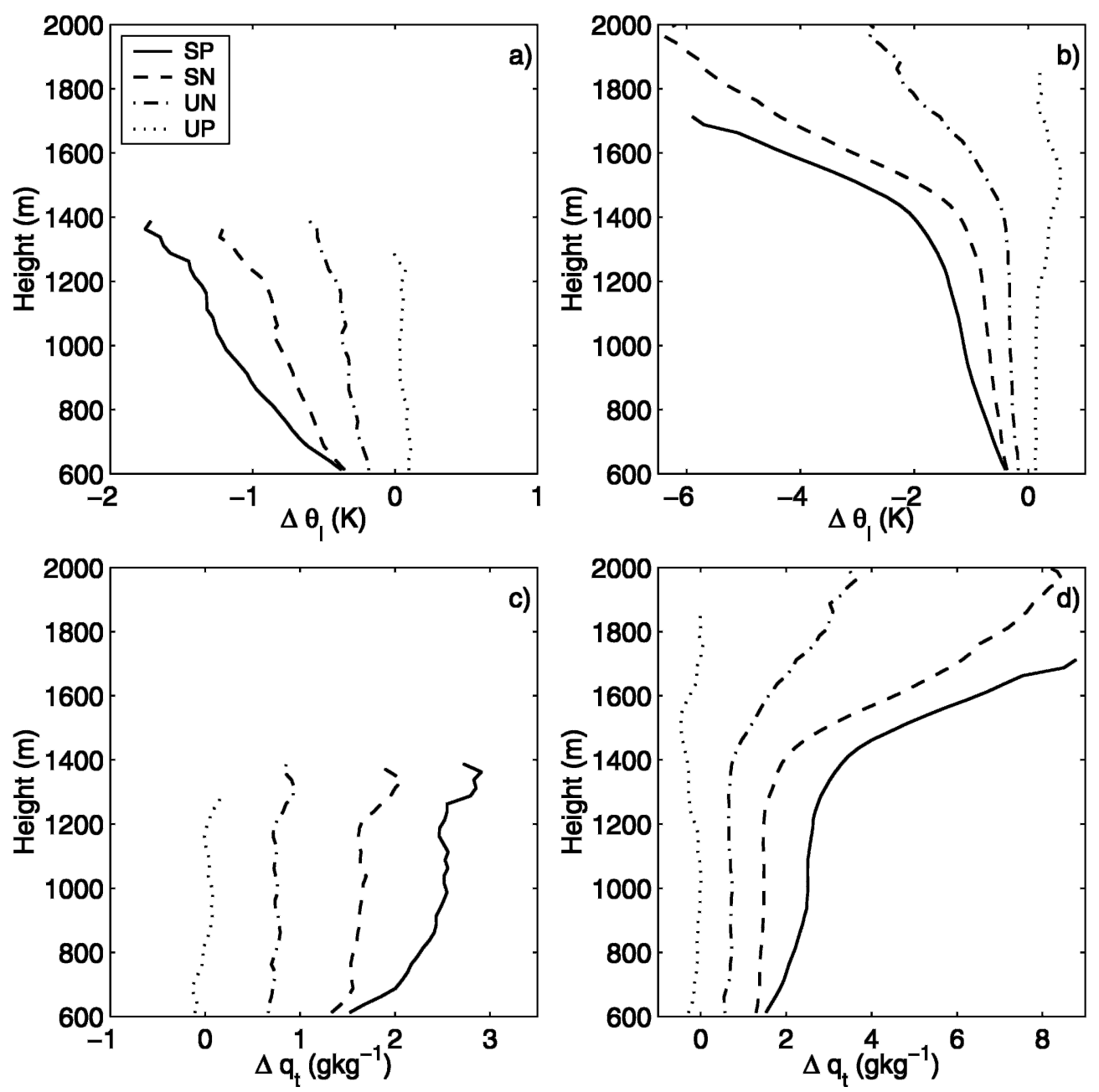

FIG. 14. (a) Vertical profiles of the $\theta_{l}$ difference between each of the four mixture categories and the environment averaged over small clouds A, B, and C. Legend labels as in Fig. 10. (b) As in (a) but averaged over large clouds D, E, and F. (c) As in (a) but for $q_{t}$. (d) As in (b) but for $q_{t}$.

that best represent the overall conserved variable transport in these clouds. However, this is not true near the cloud top for large clouds, where all saturated air becomes negatively buoyant when reaching the maximum cloud-top height.

Siebesma and Cuijpers (1995) used an LES simulation of BOMEX convection to examine how well the turbulent flux of a conserved variable $\chi \in\left\{\theta_{l}, q_{t}\right\}$ can be represented by a top-hat model of the form $\overline{w^{\prime} \chi^{\prime}}=$ $(M / \rho)\left(\chi_{c}-\chi_{e}\right)$, where $M$ is the cloud vertical mass flux, $\chi_{e}$ is the cloud environment mean, and $\chi_{c}$ is the cloud mean with cloud determined by one of three different criteria based on grid cell liquid water content $q_{c}$, vertical velocity $w$, and buoyancy $B$. They label the three criteria as the cloud decomposition $\left(q_{c}>0\right)$, the updraft decomposition ( $w>0$ and $q_{c}>0$ ) and the cloud-core decomposition ( $w>0$ and $q_{c}>0$ and $B>0$ ). Siebesma and Cuijpers found that the top-hat representation gave the best approximation to the LES flux when $\chi_{c}$ was determined by the cloud-core decomposition. The underlying reason for this can be seen in Figs. 15a-d: although the thermodynamic fluxes produced by the SN mixtures are nonnegligible, they are approximately canceled through most of the cloud layer by the UNmixtures-produced fluxes, leaving the SP mixtures as most representative of the net $\theta_{l}$ and $q_{t}$ transport.

While the SP mixtures may provide a good approximation to the net $\theta_{l}$ and $q_{t}$ fluxes, they fail to adequately represent the buoyancy flux. Figures $15 \mathrm{e}$,f shows that the buoyancy flux in the upper $1 / 3$ of both small and large clouds is dominated by UN mixtures, while the contribution from SN mixtures is very small. Therefore, including the effects of unsaturated convection is important for the representation of both the vertical mass flux and the buoyancy flux in this cloud region. We discuss the implications of this failure of the cloud-core decomposition for shallow cloud parameterizations in section $5 b$. 

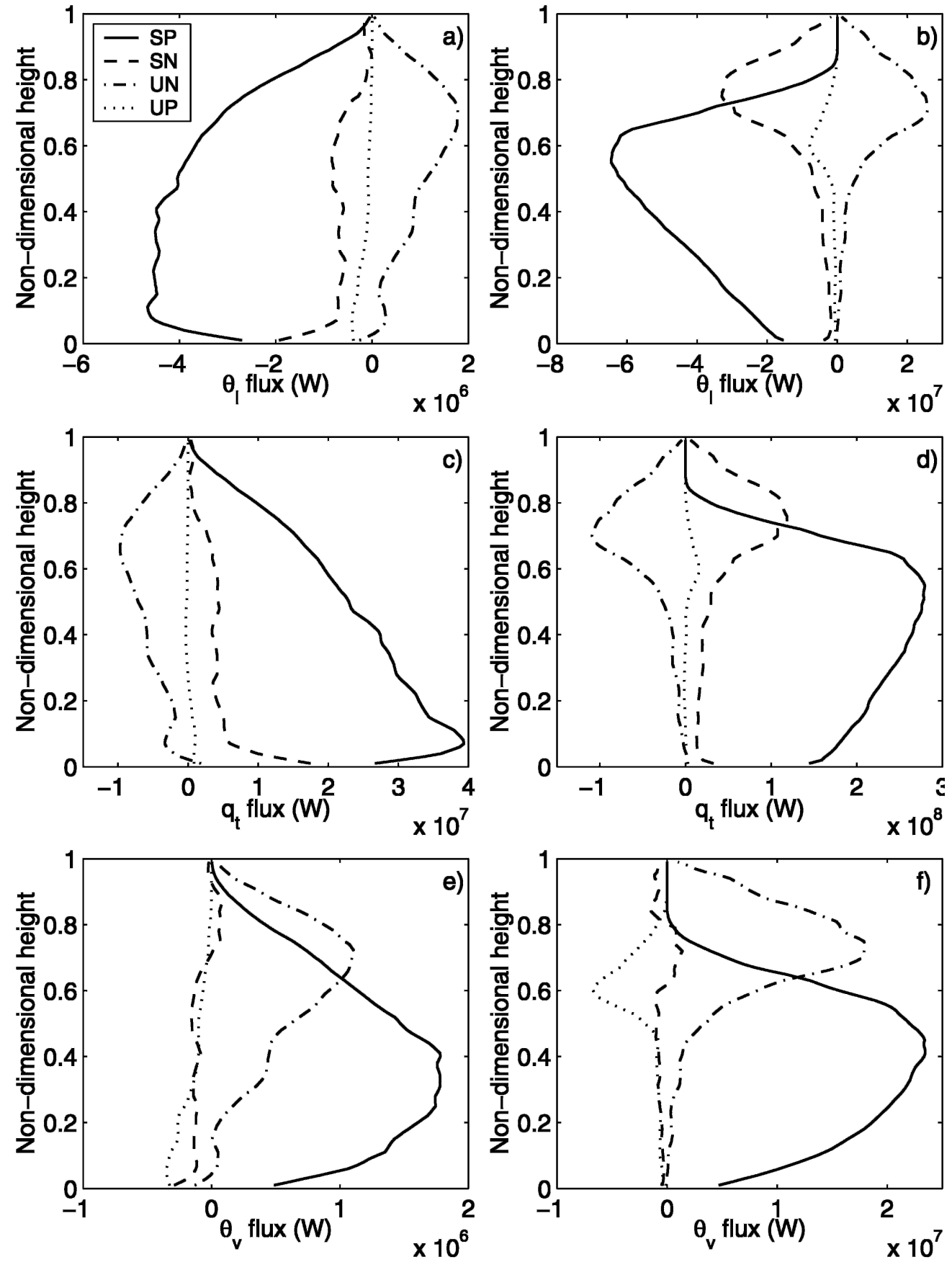

FIG. 15. Vertical profiles of $\theta_{l}, q_{t}$, and $\theta_{v}$ fluxes partitioned into contributions from the four mixture categories. Legend labels as in Fig. 10. Panels (a), (c), and (e) are averaged over small clouds A, B, and C. Panels (b), (d), and (f) are averaged over large clouds D, E, and F. Height is nondimensionalized by individual cloud height.

Figure 16 shows the total lifetime-averaged $\theta_{l}$ and $q_{t}$ fluxes for the six clouds. The $\theta_{l}$ fluxes for small clouds tend to monotonically increase with height while $q_{t}$ fluxes tend to monotonically decrease with height. Except near cloud base, this vertical distribution of the small cloud $\theta_{l}$ and $q_{t}$ fluxes indicates cooling and moist- ening of the environment throughout their cloud depth. In contrast, the large clouds tend to have minimum $\theta_{l}$ and maximum $q_{t}$ fluxes at their midlevels, indicating cooling and moistening at the upper part of cloud layer and inversion layer and warming and drying at the lower cloud layers. Note, however, that individual non- 

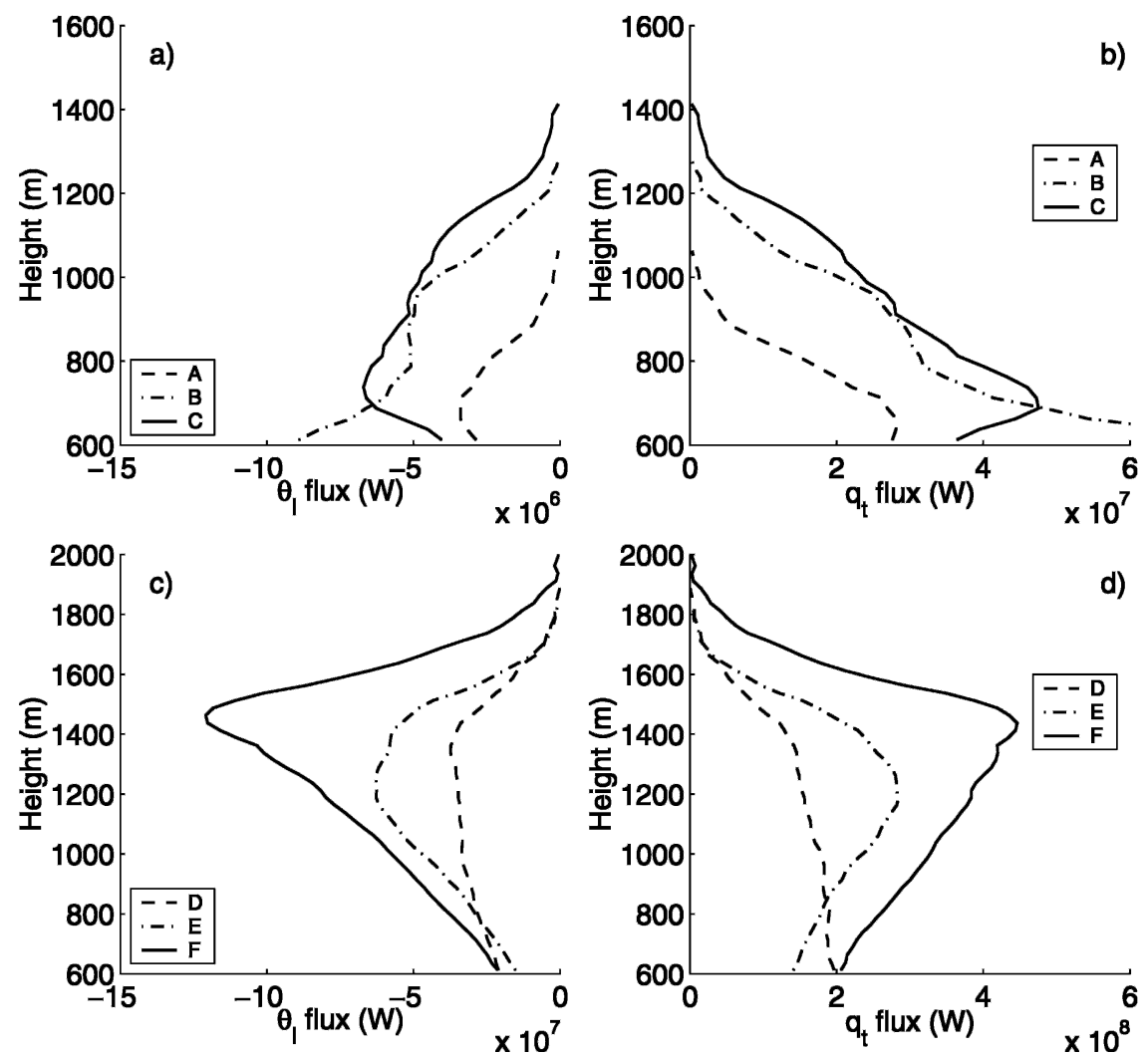

FIG. 16. Vertical profiles of (left) $\theta_{l}$ flux and (right) $q_{t}$ flux averaged over individual cloud lifetimes for clouds A-F. (top) Small clouds A, B, and C; (bottom) large clouds D, E, and F.

precipitating clouds only transport $\theta_{l}$ and $q_{t}$; the vertical integration of the convergence of $\theta_{l}$ and $q_{t}$ flux must be zero. This indicates that $\theta_{l}$ and $q_{t}$ fluxes must vanish somewhere below cloud base. Since individual clouds are only the visible part of subcloud layer thermals that are rooted in the surface layer (i.e., LeMone and Pennell 1976), this suggests that these small clouds essentially have transport behavior similar to that of the large clouds, that is, warming and drying their lower penetration depth and cooling and moistening their upper penetration depth. Here the penetration depth includes both cloud layer and subcloud layer; for the small clouds the lower penetration depth is limited to the subcloud layer.

The corresponding tendency profiles due to the transport of each cloud are shown in Fig. 17. The panels show the magnitude of the heating/cooling and drying/moistening rates found from the flux profiles of Fig. 16 assuming individual cloud fluxes extend over the model horizontal domain area $\left(6.4 \times 6.4 \mathrm{~km}^{2}\right)$. As that figure showed, small clouds cool and moisten throughout nearly their entire depth except near cloud base. The average values for these tendencies are approximately $0.02 \mathrm{~K} \mathrm{day}^{-1}$ and $0.07 \mathrm{~g} \mathrm{~kg}^{-1} \mathrm{day}^{-1}$ for the three smallest clouds. The large clouds have heating rates within the cloud layer that are roughly 10 times larger than those for the small clouds and drying rates about 5 times larger (although the net heating and drying rate of cloud D is close to zero within the cloud layer).

Within the inversion, all of the large clouds produce strong cooling and moistening with peak cooling and moistening rates of approximately $\left(0.5\right.$ to $1 \mathrm{~K} \mathrm{day}^{-1}, 1$ to $2 \mathrm{~g} \mathrm{~kg}^{-1} \mathrm{day}^{-1}$ ) near the inversion base. Comparing these values with the equilibrium large-scale forcing (e.g., ZA03) indicates that approximately four to eight large inversion penetration clouds are needed to balance the large-scale forcing within the inversion at any given time in the model domain. To counteract the cloud-base warming and drying caused by the large clouds, approximately 40 to 80 small clouds are needed. These rough estimates are consistent with the snapshots of the simulated cloud fields (not shown). In general, the differing role of small and large clouds in the cloudlayer heat and moisture budgets revealed from the LES are consistent with the results of Esbensen (1978), who combined a laterally entraining plume model to represent clouds that penetrate into the inversion with a bulk model of the shallower cloud circulation below the inversion base. He found that the deep, inversion penetrating clouds are primarily responsible for the warming and drying of the lower cloud layer, while the shal- 

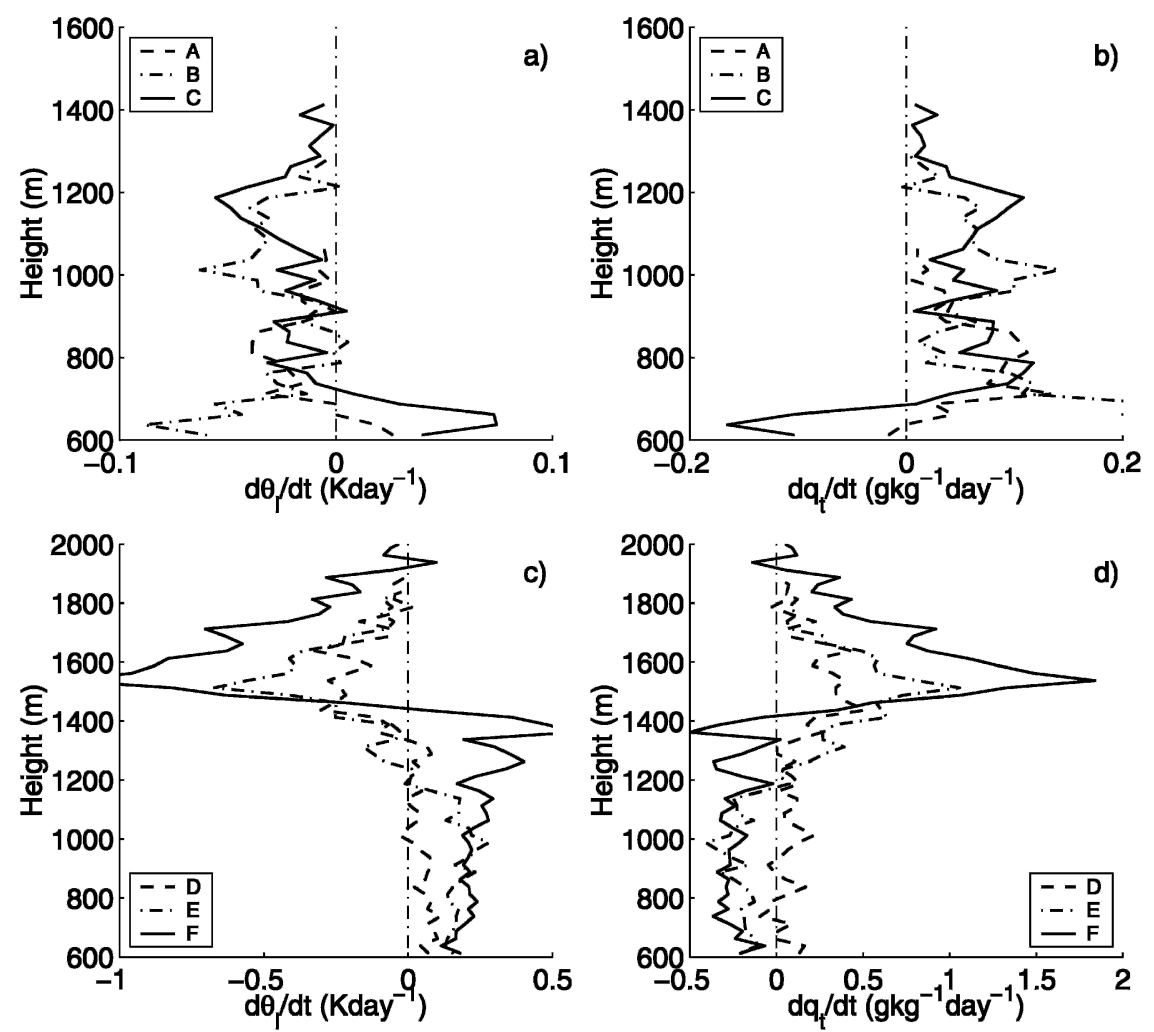

Fig. 17. Vertical profiles of environmental $\theta_{l}$ and $q_{l}$ tendencies due to the convective transport of clouds A-F over their lifetimes. (top) Small clouds A-C; (bottom) large clouds D-F. Tendencies are calculated from the vertical derivatives of the curves of Fig. 16.

lower clouds are primarily responsible for moistening below the inversion base.

\section{Sensitivity of the detrained mixed region to threshold choices}

As discussed in section $2 \mathrm{c}$, while the heightindependent threshold pair $\Delta \theta_{v, 0}=0.2 \mathrm{~K}$ and $w_{0}=0.5$ $\mathrm{m} \mathrm{s}^{-1}$ used for determining the DMR is appropriate for small clouds and for large clouds below the inversion, it is likely that it will underestimate the volume of the DMR for clouds that reach the inversion, due to additional gravity-wave-induced variability in the environment at that level. Choosing larger values of the $\Delta \theta_{v, 0}$ and $w_{0}$ thresholds would be inappropriate below the inversion where environmental buoyancy and velocity fluctuations are similar for all clouds. We would like to avoid the additional complexity of height-dependent thresholds, particularly since the error due to underestimating the volume of the DMR within the inversion (by using underestimates of $\Delta \theta_{v, 0}$ and $w_{0}$ ) should not significantly influence the net vertical transport of mass. This is because detrained air should make a negligible contribution to the net vertical mass transport, although it might strongly influence the calculated volume of the UCMR for the large clouds.

In this section we examine the impact of the choice of threshold values of $\Delta \theta_{v, 0}$ and $w_{0}$ on the UCMR volume and the UCMR vertical mass transport as a function of height. Figure 18 shows the vertical profiles of the lifetime-integrated UCMR volume and its associated vertical mass flux for the three large clouds (D, E, and F) with four different $\left(\Delta \theta_{v}, w\right)$ thresholds in the range $\left[0-1.2 \mathrm{~K}, 0-1.2 \mathrm{~m} \mathrm{~s}^{-1}\right]$. Case $2\left(\Delta \theta_{v, 0}=0.4 \mathrm{~K}, w_{0}=0.4\right.$ $\left.\mathrm{m} \mathrm{s}^{-1}\right)$ is close to the $\Delta \theta_{v, 0}=0.2 \mathrm{~K}$ and $w_{0}=0.5 \mathrm{~m} \mathrm{~s}^{-1}$ threshold used in section 3 . For this threshold, both the unsaturated/saturated volume ratios for clouds D, E, and $F(1.7,1.6$, and 1.9 , respectively) and the vertical profiles of UCMR mass flux are close to the large-cloud averaged values given in Figs. 6 and 7 . When both $\Delta \theta_{v, 0}$ and $w_{0}$ are chosen to be zero (case 1 ), all of the MR is assumed to be convective, producing a large increase in the volume of the UCMR but only a slight increase in the net downward vertical mass flux, when compared to threshold pair of case 2. This indicates that the DMR does not contribute significantly to the net vertical mass flux, and that the case 2 thresholds capture essentially all of the downward mass flux in the UCMR.

As the threshold pair is increased beyond $\Delta \theta_{v, 0}=0.4$ 

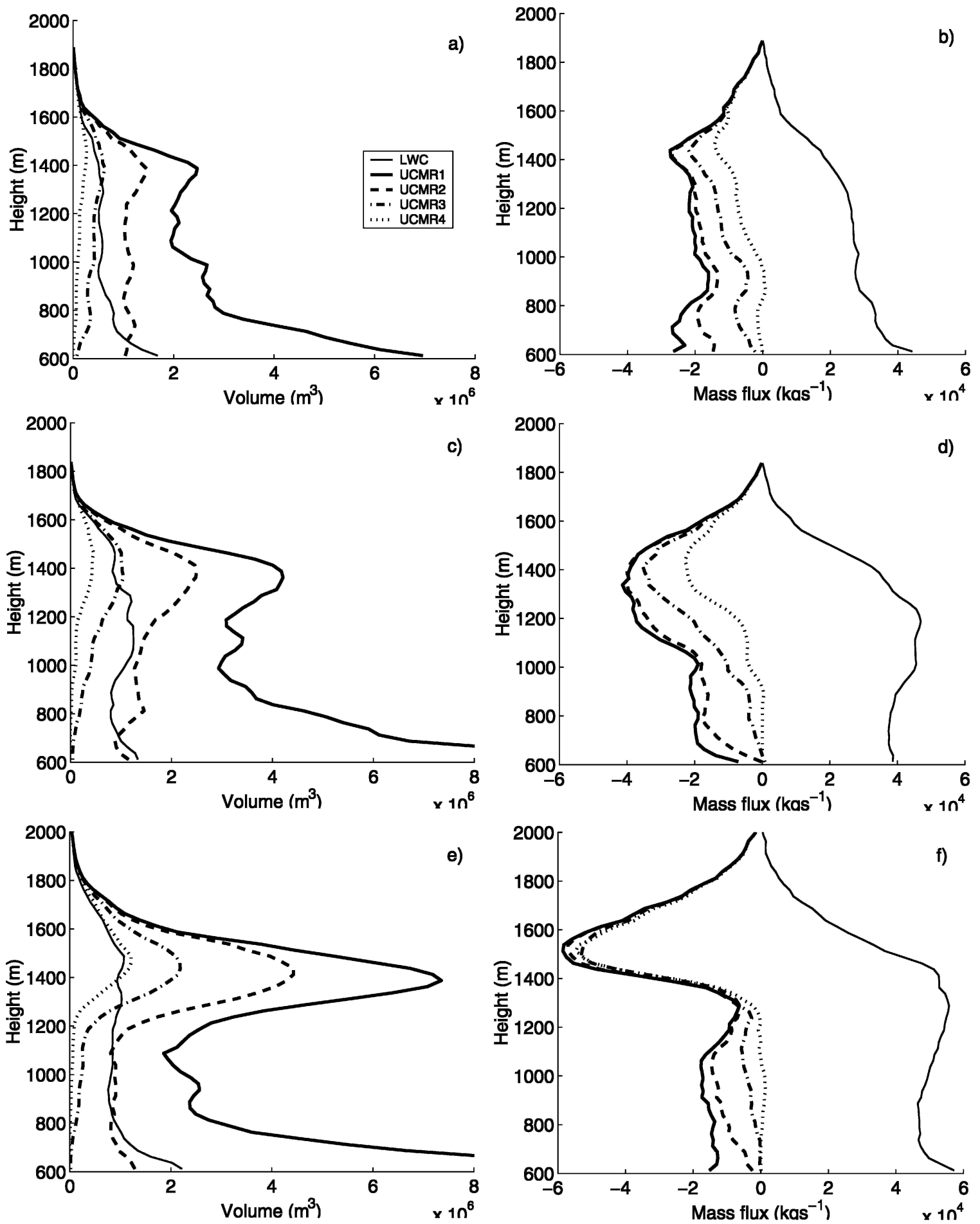

FIG. 18. Sensitivity of the volume of the UCMR and the corresponding vertical profile of the vertical mass flux to the choice of $\left(\Delta \theta_{v, 0}\right.$, $w_{0}$ ) threshold, with the LWC shown for comparison. The numbers at the top of the right panels give the ratio of the UCMR/LWC volume for each threshold choice. The threshold pairs are: 1) $\left.\left.\left(0 \mathrm{~K}, 0 \mathrm{~m} \mathrm{~s}^{-1}\right), 2\right)\left(0.4 \mathrm{~K}, 0.4 \mathrm{~m} \mathrm{~s}^{-1}\right), 3\right)\left(0.8 \mathrm{~K}, 0.8 \mathrm{~m} \mathrm{~s}^{-1}\right)$, and 4$)$ $\left(1.2 \mathrm{~K}, 1.2 \mathrm{~m} \mathrm{~s}^{-1}\right)$. (a) Vertical profile of time-integrated volume of the UCMR for cloud D. (b) As in (a) but for the corresponding vertical profile of the vertical mass flux. (c) As in (a) but for cloud E. (d) As in (b) but for cloud E. (e) As in (a) but for cloud F. (f) As in (b) but for cloud F. 
$\mathrm{K}$ and $w_{0}=0.4 \mathrm{~m} \mathrm{~s}^{-1}$ (cases 3 and 4 ), the net vertical mass flux begins to decrease, particularly below the $1500-\mathrm{m}$ level marking the base of the inversion. As these cases show, when $\left(\Delta \theta_{v}, w\right)$ thresholds are chosen to be larger than the environmental variability, there is strong sensitivity of both UCMR volume and mass flux to threshold choice. As noted in section 2c, arbitrarily large threshold values will classify all unsaturated mixtures as part of the DMR, excluding all unsaturated downdrafts and reducing the sampled CMR to only LWC.

In contrast, within the inversion the downward mass flux associated with the UCMR is insensitive to the choice of threshold value (Figs. 18b,d,f), although the UCMR volume within the inversion is significantly reduced when larger thresholds are chosen (Figs. 18a,c,e). Thus, most of the unsaturated downward mass flux within the inversion comes from a volume of UCMR that is comparable to the size of the LWC, supporting the idea that unsaturated downdrafts are confined within a small volume and are truly part of the cumulus convection. Furthermore, as shown in Fig. 6, the time evolution of the UCMR volume indicates that the UCMR and its associated downward mass flux peaks at the dissipation stage of individual clouds, indicating it is associated with cloud evaporation. In summary, the choice of threshold values made in section 2 eliminates the large majority of DMR grid cells from the CMR statistics while capturing nearly all of the convective transport both above and below the inversion.

\section{Discussion}

The vertical mass flux profile of a trade wind cumulus boundary layer results from the contributions of many individual cumulus clouds. We have used a highresolution LES to examine in detail the life cycle of six simulated clouds taken from a cloud field in equilibrium with its large-scale forcing. With the help of a passive tracer, we have partitioned the cloud life cycle transport into saturated (LWC) and unsaturated (UCMR) components and have shown that, on average, cumulus convection occurs in a region with a timeintegrated volume roughly 2 to 3 times that of the LWC. All six clouds exhibit qualitatively similar vertical mass flux profiles, with net downward motion at upper levels and net upward motion at lower levels. The results indicate that this downward mass flux comes primarily from the UCMR during the dissipation stage (Figs. 7, 8). Vertical profiles of the partitioned cloud - environment differences $\Delta \theta_{l}$ and $\Delta q_{t}$ show that the unsaturated negatively buoyant mixtures are consistently cooler and moister than the environment, indicating that the downdrafts are driven by evaporative cooling (Fig. 14). This unsaturated negatively buoyant convective mixed region provides the dominant contribution to both the buoyancy and mass fluxes in the upper portion of the cloud layer, while the saturated positively buoyant cloud mixtures dominate the fluxes at lower levels. However, small and large clouds have distinct vertical heating/cooling and drying/moistening profiles, with small clouds cooling and moistening throughout their depth while larger clouds cool and moisten at upper cloud depths and heat and dry at lower cloud depths. When the cloud vertical depth is extended to include the subcloud layer, small and large clouds show similar transport profiles; both cool and moisten their upper penetration environment and warm and dry their lower penetration environment.

\section{a. Comparison with entraining plume and EMBS models}

These LES results can be used to evaluate conceptual models of shallow cumulus clouds used for cumulus parameterizations. Most existing parameterizations are built around the idea of an entraining plume or parcel (e.g., Stommel 1947; Arakawa and Schubert 1974; Moorthi and Suarez 1992; Neggers et al. 2002). An entraining plume model represents an individual convective element or cloud using an ascending subcloud air parcel that continuously entrains environmental air with a specified entrainment rate. The entrained environmental air is homogenized instantaneously and the plume is finally detrained at its NBL. Because of this assumed entraining behavior, it always produces a monotonically increasing vertical mass flux and a concentrated cooling and moistening at a single level near an individual cloud top. In contrast, an EMBS model (e.g., Emanuel 1991; Zhao and Austin 2003) assumes that an element of subcloud air ascends adiabatically to a particular level and undergoes dilution that generates a spectrum of mixtures. These mixtures are then evaporated and vertically displaced to their individual NBLs where they are detrained into the environment. For nonprecipitating clouds, this EMBS model always produces a decreasing vertical mass flux and an extended cooling over the upper portion of individual clouds. Clearly, both conceptual models are highly simplified pictures of the transport associated with individual convective elements of real cumulus clouds.

Figure 7 shows that neither the vertical mass flux profiles of the LWC region, nor those of the CMR, are consistent with the entraining plume prediction, although the mass flux of the largest cloud F does increase slightly with height in the middle of cloud layer. Moreover, small clouds show no tendency to have larger normalized vertical mass flux; in fact their vertical mass fluxes decrease more rapidly with height. In contrast, Fig. 7 is qualitatively consistent with the EMBS model prediction, which, for shallow cumuli, always produces a decreasing vertical mass flux, with a downward mass flux dominating an individual cloud top (see, e.g., Fig. 13c in ZA03). Furthermore, Fig. 17 shows that individual clouds always produce extended cooling and moistening over a rather large fraction of individual cloud depth near their cloud top. In fact, for 
small clouds, this cooling and mostening extends over approximately their whole depth. This result is again consistent with the EMBS model (see, e.g., Fig. 9 in ZA03) and contradicts the entraining plume prediction.

\section{b. Unsaturated convection}

The simulation indicates that the decay of individual clouds or convective elements is always associated with a significant amount of downward mass transport, which begins with the collapse of the ascending turret and is further enhanced by mixing and evaporation (e.g., Fig. 8). As shown in Fig. 7, many of these downdrafts are unsaturated; they dominate the overall mass transport near the individual cloud tops. This result is consistent with the EMBS representation and is also consistent with the diagnostic results of ZA03. In ZA03, we used a BOMEX convective equilibrium sounding and the large-scale forcings to diagnose the vertical profile of the cloud-ensemble mass flux. We found that this diagnosed cloud mass flux is significantly smaller than the LWC vertical mass flux obtained from the LES in Siebesma et al. (2003). In particular, the EMBS model-diagnosed cloud vertical mass flux is downward within the inversion layer and upward within the cloud layer, while the LES LWC vertical mass flux is upward throughout both the cloud and inversion layers. We attribute this difference to the different definitions of cloud boundary in the two models. In the EMBS model, the modeled clouds include not only the LWC but also the UCMR, which can play an important role in the vertical mass transport. In contrast the cloud mass flux obtained in the LES of Siebesma et al. (2003) is calculated only for the liquid water containing grid cells. In this study, we introduce a subcloud-layer tracer into the LES to explicitly track the CMR associated with individual clouds. Figure 7 shows that by including the UCMR in the vertical mass flux calculation, all of the inversion-penetrating clouds do indeed produce net downward mass flux within the inversion.

The primary role of the convective downdrafts near individual cloud tops is to redistribute the evaporative cooling and moistening, which tends to concentrate there. Although these downdrafts have a vertical extent of only a few hundreds meters (e.g., $500 \mathrm{~m}$ for cloud F), this is a significant fraction of the individual cloud depth (e.g., $1400 \mathrm{~m}$ for cloud F). When cooling and moistening are continuously generated near cloud top, the downdrafts serve to remove this cooled air and spread it downward into a deeper layer. Therefore such transport, just like the convective updrafts induced by cloud condensational heating, is fundamentally nonlocal and, in principal, should not be represented as local mixing or direct detrainment. The fact that, for shallow nonprecipitating cumuli, all condensed water must be reevaporated over a very short life cycle emphasizes the need for a symmetrical representation of both condensational heating and evaporative cooling through their induced convective drafts. The EMBS model attempts to represent this growing phase through adiabatic lifting of subcloud air parcels and the dissipation phase through mixing, evaporation, and the descent of mixtures to their unsaturated NBLs. As shown in the partitioned mass flux (Fig. 10), for these simulated clouds the growth phase is dominated by transport associated with positively buoyant saturated mixtures, while the dominant transport in the dissipation phase is via unsaturated negatively buoyant mixtures; in both phases of the life cycle mixture transport tends to be buoyancy direct.

However, a significant amount of buoyancy-indirect transport can also be seen in Figs. 10 and 12. In particular, saturated negatively buoyant mixtures on average transport air upward while unsaturated positively buoyant mixtures on average transport air downward. This buoyancy-indirect transport is primarily associated with cloud mixture inertia and momentum mixing and may be important for large clouds at higher cloud layers. The significant amount of buoyancy-indirect transport indicates that an EMBS model, which transports every mixture based solely on its buoyancy, could potentially overestimate the buoyancy flux. This result also supports a recent parameterization approach by Bretherton et al. (2004) who modified the buoyancysorting model of Kain and Fritsch (1990) by explicitly including a fraction of negatively buoyant air into their parameterized bulk entraining and detraining updraft.

An important fact associated with this counterbuoyancy transport is that mixing continues after the individual mixtures penetrate their NBLs so that a significant number of saturated mixtures become unsaturated prior to, or just after, the transition to negative velocity. Under the usual definition of cloud, these transformed unsaturated mixtures are typically excluded from the calculation of the cloud mass flux and are treated as environmental air. The fact that the existing literature reports relatively few downdrafts in numerically simulated clouds is consistent with this mixture exclusion (e.g., Siebesma and Cuijpers 1995; de Roode and Bretherton 2003). Finite resolution and the bulk representation (all or nothing) of grid-scale saturation in numerical models tend to overestimate the rate of cloud evaporation so that some of these seemingly unsaturated mixtures may actually be saturated in real clouds. However, the dominant role of the unsaturated mixtures in this simulation strongly suggests that unsaturated downdrafts should also be an important feature of real clouds.

As shown in Fig. 15, unsaturated negatively buoyant mixtures generated by cloud penetrative entrainment of inversion air dominates the buoyancy flux in the upper one-third of the cloud depth. This upper-level buoyancy flux is missed by a cloud-core representation (e.g., Siebesma and Cuijpers 1995) leading to underestimates of the turbulent kinetic energy budget at upper levels. Capturing this portion of the layer TKE budget 
could potentially improve estimates of cloud-top entrainment in shallow cumulus parameterizations, with corresponding improvements in the estimates of transport of both water vapor and chemical species.

\section{c. Role of cloud size in convective transport}

The cumulus ensemble mass flux is modulated by both the cloud size distribution and the transport associated with individual cloud types (heights); a cumulus parameterization scheme needs to account for both (e.g., Arakawa and Schubert 1974). From a diagnostic point of view, knowing one helps to deduce the other, given the observed net effect of the cloud ensemble on the large-scale flow (i.e., the heating/cooling and drying/moistening rates). As Fig. 17 shows, the simulated large clouds cool and moisten near cloud top while heating and drying near cloud base. In contrast, small clouds tend to cool and moisten throughout most of their depth except near cloud-base level. As noted in section $3 \mathrm{e}$, this result is consistent with the diagnostic results of Esbensen (1978) and also with ZA03, which predicted the vertical profile of convective tendencies of individual clouds based on an EMBS model with a simple constant eroding rate. As discussed there, the size-differentiated vertical profile of heating and moistening can be used to understand the observed cloud size distribution and the resulting cloud ensemble transport.

The simple EMBS results of ZA03 and the more realistic simulations reported here support the following conceptual picture of equilibrium convection with a size-distributed cloud ensemble. During the adjustment toward equilibrium, smaller clouds precondition the environment by continuously cooling and moistening their upper environment. In this way, future ascending subcloud air is subject to less evaporation and becomes more buoyant and therefore is able to reach higher levels. However, taller clouds, once developed, tend to heat and dry their lower environment and therefore counteract the effect of small clouds. As shown in Fig. 17 , large clouds have heating and drying rates considerably larger than small clouds and therefore would suppress convection in a population with similar numbers of large and small clouds. If convection is to be sustained, more numerous small clouds must counterbalance the heating and drying effect of large clouds within the cloud layer. When the cloud ensemble reaches equilibrium, the heat and water vapor circulation can be described as follows: the smallest "forced clouds" (Stull 1985) feed only from subcloud layer thermals and are suppressed by all larger clouds, while clouds of intermediate size are supported by both subcloud layer thermals and the smallest clouds. In this way, the largest clouds feed from the subcloud layer not only directly from associated penetrative ascending thermals, but also indirectly from all smaller clouds. An entire population of clouds must work in concert to transport and redistribute heat and water vapor out of the subcloud layer and through the cloud layer. Thus, the cloud size distribution can be seen as the result of the equilibrium large-scale forcing and the individual cloud dynamics.

Acknowledgments. We thank Marat Khairoutdinov for making his model available and for assistance with the case setup. The simulations were primarily performed using the facilities of the Geophysical Disaster Computational Fluid Dynamics Centre at the University of British Columbia; we thank Henryk Modzelewski for Beowulf cluster computing support. The manuscript was significantly improved by the comments of Steven Esbensen, Mark Holzer, Chin-Hoh Moeng, and two anonymous reviewers. This work was supported through funding of the Modeling of Clouds and Climate Proposal by the Canadian Foundation for Climate and Atmospheric Sciences, the Meteorological Service of Canada, and the Natural Sciences and Engineering Research Council. The first author gratefully acknowledges support from a University of British Columbia University Graduate Fellowship.

\section{REFERENCES}

Arakawa, A., and W. H. Schubert, 1974: Interaction of a cumulus cloud ensemble with the large-scale environment, Part I. J. Atmos. Sci., 31, 674-701.

Betts, A. K., 1986: A new convective adjustment scheme. Part I: Observational and theoretical basis. Quart. J. Roy. Meteor. Soc., 112, 677-691.

Bretherton, C. S., and P. K. Smolarkiewicz, 1989: Gravity waves, compensating subsidence, and detrainment around cumulus clouds. J. Atmos. Sci., 46, 740-759.

_ J. R. McCaa, and H. Grenier, 2004: A new parameterization for shallow cumulus convection and its application to marine subtropical cloud-topped boundary layers. Part I: Description and 1D results. Mon. Wea. Rev., 132, 864-882.

Brown, A., and Coauthors, 2002: Large-eddy simulation of the diurnal cycle of shallow cumulus convection over land. Quart. J. Roy. Meteor. Soc., 128, 1075-1094.

Carpenter, R. L., J. K. K. Droegemeier, and A. M. Blyth, 1998: Entrainment and detrainment in numerically simulated cumulus congestus clouds. Part I: General results. J. Atmos. Sci., 55, 3417-3432.

de Roode, S. R., and C. S. Bretherton, 2003: Mass-flux budgets of shallow cumulus clouds. J. Atmos. Sci., 60, 137-151.

Emanuel, K. A., 1991: A scheme for representing cumulus convection in large-scale models. J. Atmos. Sci., 48, 2313-2335.

_ 1994: Atmospheric Convection. Oxford University Press, 580 pp.

_, and M. Zivkovic-Rothman, 1999: Development and evaluation of a convection scheme for use in climate models. $J$. Atmos. Sci., 56, 1766-1782.

Esbensen, S. K., 1978: Bulk thermodynamic effects and properties of small tropical cumuli. J. Atmos. Sci., 35, 826-837.

Grabowski, W. W., and T. L. Clark, 1993: Cloud-environment interface instability. Part II: Extension to three spatial dimensions. J. Atmos. Sci., 50, 555-573.

Grinnell, S. A., C. S. Bretherton, D. E. Stevens, and A. M. Fraser, 1996: Vertical mass flux calculations in Hawaiian trade cumulus clouds from dual Doppler radar. J. Atmos. Sci., 53, $1870-1886$.

Kain, J. S., and J. M. Fritsch, 1990: A one-dimensional entraining/ 
detraining plume model and its application in convective parameterization. J. Atmos. Sci., 47, 2784-2802.

Khairoutdinov, M. F., and D. A. Randall, 2003: Cloud resolving modeling of the ARM summer 1997 IOP: Model formulation, results, uncertainties, and sensitivities. J. Atmos. Sci., 60, 607625.

Klaassen, G. P., and T. L. Clark, 1985: Dynamics of the cloudenvironment interface and entrainment in small cumuli: Twodimensional simulations in the absence of ambient shear. $J$. Atmos. Sci., 42, 2621-2642.

LeMone, M. A., and W. T. Pennell, 1976: The relationship of trade wind cumulus distribution to subcloud layer fluxes and structure. Mon. Wea. Rev., 104, 524-539.

Lin, C., and A. Arakawa, 1997: The macroscopic entrainment processes of simulated cumulus ensemble. Part I: Entrainment sources. J. Atmos. Sci., 54, 1027-1043.

Margolin, L., P. Smolarkiewicz, and Z. Sorbjan, 1999: Large-eddy simulations of convective boundary layers using nonoscillatory differencing. Physica D, 133, 390-397.

Moorthi, S., and M. J. Suarez, 1992: Relaxed Arakawa-Schubert: A parameterization of moist convection for general circulation models. Mon. Wea. Rev., 120, 978-1002.

Neggers, R., A. P. Siebesma, and H. Jonker, 2002: A multiparcel model for shallow cumulus convection. J. Atmos. Sci., 59, $1655-1668$

Neggers, R. A. J., H. J. J. Jonker, and A. P. Siebesma, 2003: Statistics of cumulus cloud populations in large-eddy simulations. J. Atmos. Sci., 60, 1060-1074.

Nitta, T., and S. K. Esbensen, 1974: Heat and moisture budget analyses using BOMEX data. Mon. Wea. Rev., 102, 17-28.

Pope, S. B., 2000: Turbulent Flows. Cambridge University Press, $771 \mathrm{pp}$.

Raga, G., J. Jensen, and M. Baker, 1990: Characteristics of cumulus band clouds off the coast of Hawaii. J. Atmos. Sci., 47, 338-355.

Raymond, D. J., and A. M. Blyth, 1986: A stochastic mixing model for nonprecipitating cumulus clouds. J. Atmos. Sci., 43, 2708 2718.

Siebesma, A. P., and J. Cuijpers, 1995: Evaluation of parametric assumptions for shallow cumulus convection. J. Atmos. Sci., 52, 650-666.

, and A. Holtslag, 1996: Model impacts of entrainment and detrainment rates in shallow cumulus convection. J. Atmos. Sci., 53, 2354-2364.

- and H. J. J. Jonker, 2000: Anomalous scaling of cumulus cloud boundaries. Phys. Rev. Lett., 85, 214-217.

- and Coauthors, 2003: An intercomparison study for cloud resolving models of shallow cumulus convection. J. Atmos. Sci., 60, 1201-1219.

Smolarkiewicz, P., and W. Grabowski, 1990: The multidimensional positive definite advection transport algorithm: Non-oscillatory option. J. Comput. Phys., 86, 355-375.

Stevens, B., and Coauthors, 2001: Simulations of trade wind cumuli under a strong inversion. J. Atmos. Sci., 58, 1870-1891.

Stommel, H., 1947: Entrainment of air into a cumulus cloud. $J$. Meteor., 4, 91-94.

Stull, R. B., 1985: A fair-weather cumulus cloud classification scheme for mixed-layer studies. J. Climate Appl. Meteor., 24, 49-56.

_, 1988: An Introduction to Boundary Layer Meteorology. Kluwer, $666 \mathrm{pp}$.

Tao, W.-K., J. Simpson, and S.-T. Soong, 1987: Statistical properties of a cloud ensemble: A numerical study. J. Atmos. Sci., 44, 3175-3187.

Taylor, G. R., and M. B. Baker, 1991: Entrainment and detrainment in cumulus clouds. J. Atmos. Sci., 48, 112-121.

Tiedtke, M., W. A. Hackley, and J. Slingo, 1988: Tropical forecasting at ECMWF: The influence of physical parameterization on the mean structure of forecasts and analyses. Quart. J. Roy. Meteor. Soc., 114, 639-664.

von Salzen, K., and N. A. McFarlane, 2002: Parameterization of the bulk effects of lateral and cloud-top entrainment in transient shallow cumulus clouds. J. Atmos. Sci., 59, 1405-1430.

Zhao, M., 2003: Episodic Mixing and Buoyancy-Sorting Representations of Shallow Cumulus Convection. Ph.D. thesis, University of British Columbia, 219 pp.

— , and P. H. Austin, 2003: Episodic mixing and buoyancysorting representations of shallow convection: A diagnostic study. J. Atmos. Sci., 60, 892-912.

_ , and —_ cited 2004: UBC cloud physics. [Available online at http://www.eos.ubc.ca/research/clouds.]

, and 2005: Life cycle of numerically simulated shallow cumulus clouds. Part II: Mixing dynamics. J. Atmos. Sci., 62, $1291-1310$ 